\title{
Electronic properties of the Dirac and Weyl systems with first- and higher-order dispersion in non-Fermi-liquid picture
}

\author{
Chen-Huan $\mathrm{Wu}^{*}$ \\ College of Physics and Electronic Engineering, Northwest Normal University, Lanzhou 730070, China
}

July 25, 2019

\begin{abstract}
We investigate the non-Fermi-liquid behaviors of the 2D and 3D Dirac/Weyl systems with low-order and higher order dispersion. The self-energy correction, symmetry, free energy, optical conductivity, density of states, and spectral function are studied. We found that, for Dirac/Weyl systems with higher order dispersion, the non-Fermi-liquid features remain even at finite chemical potential, and they are distinct from the ones in Fermi-liquid picture and the conventional non-Fermi-liquid picture. The power law dependence of the physical observables on the energy as well as the logarithmic renormalizations due to the long-range Coulomb interaction are showed. The Landau damping of the longitudinal excitations within random-phaseapproximation (RPA) for the non-Fermi-liquid case are also discussed.
\end{abstract}

Keywords: non-Fermi-liquid behavior; Dirac semimetal; multi-Weyl semimetal; selfenergy correction; longitudinal excitations.

\section{Introduction}

Different to the normal metals where the Fermi-liquid theory is valid and the long-range Coulomb interaction can be ignored due to the static screening by the particle-hole excitations [1], for the topological insulator or when near the quantum critical in modern condensate matter physics, the non-Fermi-liquid theory is required. In Fermi-liquid theory, the excitations near the Fermi surface (usually within the order parameter fluctuation gap) are Fermionic, that results in the uniform spin susceptibility[2] in contrast to the one in topological insulator [3], and also leads to the linear-temperature-dependence of the electronic specific heat rather that the logarithmic divergent one as found in heavy-fermion system as well as the superconductors. While at quantum critical point, the order paramater quantum fluctuation is a gapless boson mode, and leads to the non-Fermi-liquid behavior by coupling to the gapless Fermionic excitations. Such coupling is accompanied by a strong Landau damping, and with the small quasiparticle residue and large effective mass [4, 5, 6], which can be obtained from the self-energy and dispersion. As the strong fluctuation, e.g., in strongly correlated systems, is presented, the fixed point start from Fermi liquid in renormalization group flow is destabilized. In this letter, we investigate the non-Fermi-liquid behaviors of the 2D Dirac system with first-order dispersion in continuum model. The exchange-induced Fermionic and Bosonic self-energy correction as well as other observable quantities are calculated and also for these of the Dirac/Weyl systems with higher dispersion. Considering the disorder effect origin from the impurities, the polaron

*chenhuanwu1@gmail.com 
as a excited quasiparticle are important when consider the many-body effect (many-electron effect), and the disorder-induced self-energy[7] describes the impurity-Fermions (for Fermionic polaron) or impurity-Bosons (for Bosonic polaron) interaction and with the impurity dressed by the corresponding particle-hole excitations. Besides, since the spin rotation is missing in the Dirac $\delta$-type impurity field, the spin structure is fixed, and the spin of impurity and that of majority particles are usually opposite, that provies the opportunity to form the Cooper pair and the strongly bound dimer.

In the usual Dirac or Weyl systems, the proportion of the interacting electron keeps increasing as the energy is lowered down to zero along the cone-like dispersion, and finally at the zeroenergy point, the noninteracting electrons still exist. At higher energy, the umklapp scattering can open up a local charge gap at the middle point of Brillouin zone edge, i.e., the $M$-point, and leads to a symmetry-breaking instability to breaks the Landau-Fermi liquid picture. This can be implemented by the magnetic-doping [8, 9], which is in constract to the non-magnetic doping [4]. For high non-magnetic doping [8, 10] (with strong short-range interaction), the saddle points are lifted above the Fermi level, and the effect of umklapp scattering is being lessened, thus in such nonadiabatic case, the interaction (short-range) strength increases and induces some kinds of pairing instabilities like the $d$-wave superconductivity, which will not breaks the Fermi-liquid picture. In the mean time, the nonadiabaticity leads to lower Fermi velocity. This is in contrast to the case of long-range electron-electron interaction which is nearly unscreened in the charge neutrality point of the undoped Dirac or Weyl systems [11], where the conventional Landau theory is inapplicable, and the self-energy and Fermi velocity can be logarithmically enhanced[11, 12]. We have also obtain[4] that, the large gap in $M$-point (which becomes largest at zero doping [10]) also supports the adiabatic feature of the transport of electrons through the Dirac cone or Weyl cone, which corresponds to the non-Fermi-liquid picture. This is also consistent with the GW calculation results 10] that the gap in $M$-point increases when the doping level closes to zero (i.e., the adiabatic non-Fermi liquid regime). It is shown that even at low temperature where the umklapp scattering can be ignored, the non-Fermi-liquid with very high resistivity can still appears by aid of the strong electron-acoustic phonon coupling[13] which also brings the superconducting instability.

The Fermi-liquid to non-Fermi-liquid transition also affects the plasmon dispersion. In Fermi-liquid picture with finite doping, the short-range Hubbard-like interaction has both the density and spin channels with the charge-density susceptibility and spin-density susceptibility. The induced spin plasmon frequency acts as $\omega_{p} \sim \sqrt{q}$ for $q \ll k_{F}$ and $\sim \alpha q$ for $q \gtrsim k_{F}$ ( $q$ is the scattering wave vector), which is widely seen in the ordinary 2D Fermi-liquid. In this regime, the dimensionless effective fine structure constant (Wigner-Seitz radius[14]) $r_{s}=e^{2} / \hbar v_{F} \epsilon$ is small due to the strongly screened Coulomb potential (in the limit of $\mu \gg \omega$ and $q \ll k_{F}$ ), and thus the method of RPA provides an accurate result. That can also be seen from the relation $r_{s} \propto 1 / \sqrt{n}$ in $2 \mathrm{D}$ system, which shows that the Coulomb interaction decreases with the raising doping level. In the mean time, the gap in $M$-point as well as the fermi velocity decreases[11], and the phenomenon of Pauli blocking emergents during the interband transitions. Note that in this limit, the transverse spin fluctuation, if exist, will much stronger than the charge density fluctuation (with longitudinal excitations), for example, in Helical liquid[15] or even the chiral liquid system[16, 17] like magnetic graphene edges[18]. While for the non-Fermi-liquid picture in the zero-doping limit, the preserved long-range Coulomb interaction has only the charge channel, and the accuracy of RPA decreases with the increasing $r_{s}$. The Coulomb potential is then obtained by 15$]=2 \pi \hbar v_{F} r_{s} / q \sim r_{s}$. In this case, if we focus on the low-energy regime with small scattering momentum $q$ and ignore the electron-phonon interaction, the RPA susceptibility still gives the plasmon mode which $\sim \sqrt{q}[15]$ and the system is still adiabatic, however, if we takes the phonon umklapp scattering into account and considering the momenta outside the first Brillouin zone which with higher energy, the nonadiabatic feature will becomes 
more obvious with the phonon frequency $\Omega$ larger than Fermi energy $E_{F}$, and that results in a quasilinear polaronic plasmon mode which behaves as $\sim \beta q$ (here $q$ is the phonon scattering momentum and $\beta \ll \alpha$ ). This has been experimental observed[19, 9, 20] in the topological insulators. In the mean time, the phonons decreases the group velocity of electrons near the nodes, and exhibit nonadiabatic feature [4, and the quasiparticle resideu $Z$ also becomes lower in these nodes. It is interesting that, even in the absence of phonon umklapp scattering (at lowenergy regime), the strong electron-phonon coupling could still breaks the Fermi-liquid picture. That is because the strong many-body interaction effects can drive the 2D metallic Fermi liquid ground state into gapped insulator phase as the strong correlation goes beyond the Fermi-liquid paradigm 13, 5. Similar phenomenon can also be realized by the topological quantum phase transition through the merger of Weyl nodes with opposite chirality [21, 22, 23]. Such a largely increased resistivity with the breakdown of Fermi-liquid picture has been observed recently in the (AA-stacked) twisted bilayer graphene near the magic angle 24, 13, (at metallic phase) and the cuprates. Such strange metallic behavior is been ascribed to the strong electron-acoustic phonon interaction accompanied with the flattening of conduction band and valence band near the cone. With the increace of electron-phonon interaction, the slope of band is lowered and thus the Fermi velocity of electron also decrease. That also leads to the nonadiabatic effects with the enhanced interaction strength, like the decrease of quasiparticle residue $Z$ and increase of effective mass (not the Dirac mass). While at low-temperature limit, similar effect could also be observed (even more obvious) in the Weyl system with high-order dispersion. It is because the dispersion at Dirac or Weyl cone has a decisive effect on the long-range Coulomb interaction.

In this paper, we consider the case of low- $q$ (without the internode scattering), and ignore the electron-phonon coupling as well as the possible induced short-range electron-electron attraction 25]. The differences maked by the high order dispersion can be showed by the topological nature as well as the property of spin-momentum locking[21], also, the electronic properties like the self-energy and optical conductivity also modified by it. The density-ofstates (DOS) in the low-energy limit will be largely increased when the dispersion order $m$ turns up, and accompanied with the enhanced dissipation of energy. This may be the reason of the vanishing of residue $Z$ in low-energy limit of the multi-Weyl semimetals with high-order dispersion, which is impossible for the linear Dirac or Weyl dispersion[30], although the screening to electron-electron interaction is heavily suppressed in neutral point of the undoped intrinsic Dirac or Weyl systems as the DOS goes to zero. We note that for 1D Luttinger liquid, the compressibility will not vanishes even in zero residue[26]. In Sec.2, we discuss the selfenergy correction in 2D Dirac system. In Sec.3, we discuss the disorder effect and free energy. The expressions of self-energy, optical conductivity and DOS of the Dirac and Weyl systems characterized by the high order dispersion are analytically derived in the Sec.5.

\section{Self-energy correction in 2D Dirac system}

The isotropic $(2+1)$ D Dirac Fermions coupled to long-range Coulomb interaction can be described by the effective action

$$
S=\int d \tau d^{2} r\left\{\psi^{\dagger}\left[\partial_{\tau}-i g \phi+H_{0}(k)\right] \psi+\frac{1}{2}\left[\left(\partial_{x} \phi\right)^{2}+\left(\partial_{y} \phi\right)^{2}\right]\right\}
$$

where $\psi$ is the Fermion field and $\phi$ is the Bosonic field which describes the long-range instantaneous Coulomb interaction and related to the order parameter, and the Fermions couples to the Bosons through the coupling constant $g^{2}=2 \pi e^{2} / \epsilon>0$ where $\epsilon$ is the background dielectric constant. Here we focus on the long-range Coulomb interaction, while for the doped case with strong fluctuation[], the strong attraction within the short-range pairs may induces the Mott 
insulator phase [27, 28] or the superconductivity order parameter at finite doping[1]. The $H_{0}(k)$ is the non-interacting Hamiltonian which for 2D linear Dirac system reads

$$
H_{0}(k)=\hbar v_{F}\left(\eta k_{x} \tau_{x}+k_{y} \tau_{y}\right)+D s_{z} \tau_{z}-\mu
$$

whose eigenvalues can be obtained by solving $\operatorname{det}(H-E)=0$ as

$$
\begin{aligned}
E_{k \pm} & = \pm \sqrt{\hbar^{2} v_{F}^{2} k^{2}+D^{2}+\mu^{2}-2 \eta \hbar v_{F} k \mu \cos \theta}-\mu \\
& \approx \pm \sqrt{\hbar^{2} v_{F}^{2} k^{2}+D^{2}}-\mu,
\end{aligned}
$$

where $\theta=\arctan \frac{k_{y}}{k_{x}}$. By defining the scattered momentum as $k^{\prime}=k+q$, the exchange-induced Fermion self-energy is given by

$$
\Sigma(q)=-g^{2} \int \frac{d \Omega}{2 \pi} \frac{d^{2} k}{(2 \pi)^{2}} G_{0}\left(\Omega+i 0, k^{\prime}\right) D_{0}(k),
$$

and it's independent of the Boson Matsubara frequency $\omega=2 m \pi T$ due to the instantaneous approximation of the Coulomb interaction in one-loop order. The instantaneous Coulomb interaction is given by the scalar potential which has the propagator

$$
\begin{aligned}
\left\langle T \phi(t, \mathbf{r}) \phi\left(t^{\prime}, \mathbf{r}^{\prime}\right)\right\rangle & =-i \delta\left(t-t^{\prime}\right) \frac{1}{2} \int \frac{d^{2} k}{(2 \pi)^{2}} e^{i \mathbf{k} \cdot\left(\mathbf{r}-\mathbf{r}^{\prime}\right)} \\
& =\frac{\delta\left(t-t^{\prime}\right)}{4 \pi\left(r-r^{\prime}\right)}
\end{aligned}
$$

and that leads to the nonrelativistic features with the broken Lorentz invariance, as widely seen in the non-perturbative RG analysis [29] (while in perturbative RG analysis the instantaneous approximation is sometimes unadopted due to the effect of vector potential[30]). $G_{0}(\Omega+$ $\left.i 0, k^{\prime}\right)=\frac{1}{\Omega+i 0-H_{0}}$ is the bare Green's function (Fermion propagator). The infinitesimal quantity $i 0$ (corresponds to the scattering rate or the Fermionic damping rate) is important for the convergence of the integral and its sign is the same as that of the frequency (here we assuming the positive frequency). The Pauli exclusion principle also enforces $i 0 \rightarrow 0$ in the static limit. $D_{0}(k)=1 / k$ is the bare Boson propagator. Thus for static case, we have for a single Fermion species

$$
\begin{aligned}
\Sigma(q)= & -\frac{g^{2}}{(2 \pi)^{2}}\left[\operatorname{Li}_{2}\left(\frac{-D+\hbar v_{F} \Lambda+\mu+\Omega+i 0}{\hbar v_{F} \Lambda}\right)-\operatorname{Li}_{2}\left(-\frac{-D-\hbar v_{F} \Lambda+\mu+\Omega+i 0}{\hbar v_{F} \Lambda}\right)\right. \\
& +\ln \left(D-\hbar v_{F} \Lambda-\mu-\Omega-i 0\right) \ln \left(-\frac{-D+\mu+\Omega+i 0}{\hbar v_{F} \Lambda}\right) \\
& \left.-\ln \left(D+\hbar v_{F} \Lambda-\mu-\Omega-i 0\right) \ln \left(\frac{-D+\mu+\Omega+i 0}{\hbar v_{F} \Lambda}\right)-(\ln (-\Lambda)-\ln \Lambda) \ln (-D+\mu+\Omega+i 0)\right]\left.\right|_{\Omega}
\end{aligned}
$$

where $\operatorname{Li}_{2}(z)=\sum_{k=1}^{\infty} \frac{z^{k}}{k^{2}}$ is the dilogarithm function. Unlike the momentum shell integration, we only applied the ultraviolet cutoff here to deal with the non-Fermi liquid with nearly zero gap (and chemical potential). Ultraviolet cutoff during the calculation is important to prevent the divergence of integral. At higher temperature, the repulsive Coulomb interaction is competes with the attractive electron-phonon coupling. The unscreened on-site Coulomb repulsion averts the double occupation of the lattice sites and thus closing the gap, while the electron-phonon coupling is opposite. The lowest-order contribution to the exchange-induced self-energy reads

$$
\Sigma(k, \omega)=T \sum_{\Omega} \int \frac{d^{2} q}{(2 \pi)^{2}} U V(q, \Omega) G(k-q, \omega-\Omega),
$$


where $U$ is the Coulomb repulsion potential and

$$
V(q, \omega)=-\int \frac{d^{2} q}{(2 \pi)^{2}} G(k, \Omega) G\left(k^{\prime}, \Omega^{\prime}\right)
$$

is the fluctuation exchange potential. Here we approximate the irreducible vertex function to the on-site Hubbard interaction, and the resulting exchange self-energy is obviously beyond the instantaneous approximation

while for the attractive phonon-mediated interaction, similarly, the self-energy reads

$$
\Sigma(k, \omega)=T \sum_{\Omega} \int \frac{d^{2} q}{(2 \pi)^{2}} U_{e-p h} P(q, \Omega) G(k-q, \omega-\Omega),
$$

with the phonon propagator (in lowest-order) [31]

$$
P(q, \Omega)=\frac{\Omega_{p h}^{2}}{\Omega_{p h}^{2}+\Omega^{2}}
$$

where $U_{e-p h}$ is the electron-phonon coupling parameter and $\Omega_{p h}$ is the phonon frequency. For strong enough on-site attractive Hubbard interaction, the charge-density-wave (CDW) phase or the gapless semimetal phase will be unstable to the s-wave superconducting phase, and thus the symmetry described by $\left\langle\psi_{+}\left|\sigma_{x / y}\right| \psi_{-}\right\rangle=0$ is broken ( \pm refers to the up and down spin respectively) which don't consider the orbital degree of freedom. For 2D Dirac semimetal, due to the absence of the impurity scattering in the Dirac point with zero density of states, the short-range interaction is weak and insufficient to destabilize the Dirac Fermions. For superconducting phase without the Coulomb repulsion (instantaneous Coulomb interaction) and the disorder, the Lorentz invariance is possible with isotropic Fermion and Boson velocities (i.e., in case of the supersymmetry which interchanges bosons and fermions[32]), which can be realized at low-energy by a metallic (polarizable) superstrate. Through the minimum model in Eq.(2), the time-reversal symmetry can be shown as

$$
\Theta H_{0}(k) \Theta^{-1}=\tau_{y} H_{0}^{*}(k) \tau_{y}=\hbar v_{F}\left(-\eta k_{x} \tau_{x}-k_{y} \tau_{y}\right)+D \tau_{z} \sigma_{z}-\mu=H_{0}(-k),
$$

where $\Theta=i \tau_{y} K$ is the time-reversal operator. While for the $2 \mathrm{D}$ lattice model

$$
H_{0}^{l}(k)=\sum_{k} c_{k}^{\dagger}\left[t \sin k_{x} \tau_{y}+d_{z}(k) \tau_{z}\right] c_{k}-\sum_{k} c_{k}^{\dagger} \mu c_{k},
$$

where $d_{z}(k)$ is the momentum-dependent gap function. For this lattice model, the particle-hole symmetry at half-filling can be revealed by

$$
\Xi H_{0}^{l}(k) \Xi^{-1}=\tau_{y} H_{0}^{l *}(k) \tau_{y}=\sum_{k} c_{k}^{\dagger}\left[-t \sin k_{x} \tau_{y}-d_{z}(k) \tau_{z}\right] c_{k}=H_{0}^{l}(-k),
$$

where $\Xi=\tau_{y} K$ is the particle-hole operator. Topologically, $\Theta^{2}=0, \pm 1$ corresponds to the time-reversal symmetry and $\Xi^{2}=0, \pm 1$ corresponds to the particle-hole symmetry. Although the time-reversal symmetry and the inversion symmetry are broken in the presence of gap function or by the charge-density-wave (CDW) order formed by polarized electrons, the symmetry described by the product of $\Theta$ and the in-plane mirror reflection operator $M_{x}$ could be preserved[33], i.e., $\Theta M_{x}$ which protect the semimetal nature against the weak short-range interaction. The weak short-range interaction can't be taken into account the RG analysis, while the frequency-dependent self-energy in a non-Fermi liquid system is proportional to the anomalous dimension and the RG parameter (the logarithmic term). The anomalous dimension 
also implies the missing of the pole struture of the Green's function, which correspond to the electron addition and removal energies in the noninteracting case 34.

In one-loop order, the bare Boson propagator (phonon or the photon) is modified as $D(\Omega, k)=$ $\left(k^{2}-\Sigma_{b}(\Omega, k)\right)^{-1}$ where $\Sigma_{b}(\omega, k)$ is the Boson self-energy in density-density correlation form:

$$
\begin{aligned}
\Sigma_{b}(\omega, q) & =g_{s} g_{v} T g^{2} \int \frac{d \Omega}{2 \pi} \frac{d^{2} k}{(2 \pi)^{2}} \operatorname{Tr}\left[\sigma_{0} G_{0}\left(\Omega^{\prime}, k^{\prime}\right) \sigma_{0} G_{0}(\Omega, k)\right] \\
& =g_{s} g_{v} T g^{2} \int \frac{d \Omega}{2 \pi} \frac{d^{2} k}{(2 \pi)^{2}} 4 \frac{\left(-\Omega^{\prime}-2 i 0-\mu\right)(-\Omega-i 0-\mu)+k k^{\prime}+D^{2}}{\left[\left(-\Omega^{\prime}-2 i 0-\mu\right)^{2}-\left(k^{\prime 2}+D^{2}\right)\right]\left[(-\Omega-i 0-\mu)^{2}-\left(k^{2}+D^{2}\right)\right]} \\
& =g_{s} g_{v} g^{2} \frac{1}{2 \pi} \int \frac{d^{2} k}{(2 \pi)^{2}} \frac{1}{4} \sum_{s s^{\prime}}\left(1+s s^{\prime} \frac{k k^{\prime}+D^{2}}{\varepsilon_{k} \varepsilon_{k^{\prime}}}\right) \frac{N_{F}\left(s \varepsilon_{k}\right)-N_{F}\left(s^{\prime} \varepsilon_{k^{\prime}}\right)}{s \varepsilon_{k}-s^{\prime} \varepsilon_{k^{\prime}}+\omega+i 0},
\end{aligned}
$$

where we use the discrete values of the frequency since otherwise the above integral becomes zero, and the formula

$$
T \sum_{\Omega} \frac{1}{-\Omega-i 0-\mu+\varepsilon_{k}} \frac{1}{-\Omega^{\prime}-2 i 0-\mu+\varepsilon_{k^{\prime}}}=\frac{N_{F}\left(\varepsilon_{k}\right)-N_{F}\left(\varepsilon_{k^{\prime}}\right)}{\varepsilon_{k}-\varepsilon_{k^{\prime}}+\omega+i 0}
$$

is used. Here $\Omega^{\prime}=\Omega+\omega$ and $g_{s} g_{v}=2$ denotes the Fermion species (spin and valley degrees of freedom). $N_{F}(x)=\left(1+e^{x / T}\right)^{-1}$ is the Fermi-distribution function. The above expression also implies that the Boson self-energy is related to the equation of motion for the bare Green's function. Through the Ward identity $\gamma=\partial_{\omega} \Sigma$ which is independent of both the external frequency and the scattering wavevector, the vertex function can be obtained as

$$
\gamma=-\frac{g^{2}}{2} \int \frac{d \Omega}{2 \pi} \frac{d^{2} q}{(2 \pi)^{2}} \operatorname{Tr}\left[\sigma_{0} G_{0}(\Omega, q) \sigma_{0} G_{0}(\Omega, q)\right] \frac{1}{q^{2}-\Sigma_{b}(\Omega, q)} .
$$

To simplify the calculation, we restrict ourselves to the gapless case, then the Boson self-energy becmoes

$$
\begin{aligned}
\Sigma_{b}(\Omega, q)= & g_{s} g_{v} g^{2} \frac{1}{(2 \pi)^{2}} \int k d k \frac{1}{4}\left(1-\frac{k k^{\prime}}{\varepsilon_{k} \varepsilon_{k^{\prime}}}\right) \frac{1}{\varepsilon_{k}+\varepsilon_{k^{\prime}}-\Omega-i 0} \\
= & g_{s} g_{v} g^{2} \frac{1}{(2 \pi)^{2}} \frac{\hbar^{2} v^{2}-1}{16 \hbar^{4} v^{4}}\left\{\left[\left(-\hbar v_{F} q+\Omega+i 0\right) \ln \left(-\hbar v_{F}(2 \Lambda+q)+\Omega+i 0\right)+2 \hbar v_{F} \Lambda\right]\right. \\
& \left.-\left[\left(-\hbar v_{F} q+\Omega+i 0\right) \ln \left(-\hbar v_{F} q+\Omega+i 0\right)\right]\right\}
\end{aligned}
$$

where $s s^{\prime}=-1$ here due to the dominating interband transition. Then the vertex function at half-filling is

$$
\begin{aligned}
\gamma= & -\frac{g^{2}}{2(2 \pi)^{2}} \int d \Omega d q \frac{q}{-\hbar v_{F} q+\Omega+i 0} \\
& {\left[q^{2}-2 \alpha \hbar v_{F} \Lambda+\alpha\left(-\hbar v_{F} q+\Omega+i 0\right) \ln \left(-\hbar v_{F} q+\Omega+i 0\right)\right.} \\
& \left.-\alpha\left(-\hbar v_{F} q+\Omega+i 0\right) \ln \left(-\hbar v_{F}(q+2 \Lambda)+\Omega+i 0\right)\right]^{-1} .
\end{aligned}
$$

\section{Disorder effect and free-energy in grand-canonical ensemble}

For the disordered system, the Fermion self-energy in lowest-order approximation reads $\Sigma^{L O}=n_{i} V_{k, k^{\prime}} / \hbar$ where $n_{i}$ is the impurity concentration and $V_{k, k^{\prime}}$ is the scattering potential. For localized potential, the disorder-induced self-energy could be momentum-independent due to the rotational invariance. In low-order perturbation theory, the self-energy matrix contains a gap function in the diagonal element, while the non-diagonal elements are missing. 
The random-phase approximation (RPA) results is valid only in the long-wavelength limit as well as the low-energy limit for large flavor number analysis, in which case the Eliashberg theory as well as the Migdal's theorem are valid. In this case the Boson propagator (as well as the Boson-frequency-related spin susceptibility) is overdamped due to the small Boson velocity and small external Boson momentum (compared to the Fermionic ones) with the Landau damping. The above results are correct for the low-energy Fermions excitations (within the band gap) for RPA which with chemical potential much larger than $k_{B} T$. Inversely, the non-Fermi-liquid feature emerges for the case of $\mu<k_{B} T$. The strong screeing effect by polarized Fermions to the disorder also provides the possibility to recover the Fermi liquid within the spectrum gap of the order parameter fluctuations (of the order of $D^{2} / W$ where $W$ is the Fermion bandwidth) which with coherent Bosonic excitations except when the disorder-induced linewidth[35] is larger than the excitation energy. In the mean time, the excitation gap gives rise to the dissipation effect which is related to the free energy and the conductivity. Further, the response function is nonzero even at $q=0$ for the Bosonic frequency in the range $\omega<v_{F} q<2 D<2 \mu$. While for the Bosonic frequency $\Omega$ larger than $v_{F} q$, the transverse spin excitation (still within the band gap) is the Goldstone spin wave and thus it's gapless in the long-wavelength limit, in contrast to the longitudinal excitations which is gapped even at $q=0$.

Unlike the weak short-range interaction, the electron-electron interaction mediated by the gapless Bosonic mode is long ranged at the quantum critical point and with the gapless critical fluctuation of the order parameter (about the Bosonic excitations) which can be described by the Ginzburg-Landau function. The Ginzburg-Landau function (the free energy) here describing the order parameter fluctuation does not contains the term $\phi^{*} \partial_{\tau} \phi$ due to the particle-hole symmetry as state above, where $\phi$ is the two component complex amplitude. For bipartite system, the particle-hole symmetry suggests the exitence of the zero-energy modes which satisfy $\phi_{A}= \pm i \phi_{B}$. In the presence of particle-hole symmetry the ac Hall conductivity vanishes while the dc conductivity is preserved[36]. At finite temperature, the free energy can be obtained by the following partition function base on the Fermion propagator as

$$
\mathcal{Z}=\operatorname{det}\left[\frac{1}{G_{0}(k, \Omega) T}\right]=\prod_{\varepsilon}\left(\frac{\Omega+i 0-H_{0}}{T}\right)_{\varepsilon}^{2},
$$

then the free energy density reads

$$
F=-T \int \frac{d^{2} k}{(2 \pi)^{2}} \sum_{i \Omega=0}^{n} \ln \mathcal{Z}=-T \lim _{n \rightarrow \infty} \int \frac{d^{2} k}{(2 \pi)^{2}} \ln \left[\frac{H_{0}^{2}}{T^{2 n+2}}\left(\frac{\Gamma\left(1-H_{0}+n\right)}{\Gamma\left(1-H_{0}\right)}\right)^{2}\right] .
$$

The above integral can be analytically solved as

$$
\begin{aligned}
F= & -T \lim _{n \rightarrow \infty} \frac{1}{2 \pi 2 \hbar^{2} v_{F}^{2}}\left[-2 D^{2} \ln \left(H_{0}\right)-2 \hbar^{2} v_{F}^{2} k^{2} \ln \Gamma\left(-H_{0}+n+1\right)+2 \hbar^{2} v_{F}^{2} k^{2} \ln \Gamma\left(-H_{0}+1\right)\right. \\
& +\hbar^{2} v_{F}^{2} k^{2} \ln \left(\frac{H_{0}^{2}}{T^{2 n+2}}\left(\frac{\Gamma\left(-H_{0}+1+n\right)}{\Gamma\left(-H_{0}+1\right)}\right)^{2}\right)-4 \psi^{(-3)}\left(-H_{0}+n+1\right)+4 \psi^{(-3)}\left(-H_{0}+1\right) \\
& -4 \hbar v_{F} k \psi^{(-2)}\left(-H_{0}+n+1\right)+4 \hbar v_{F} k \psi^{(-2)}\left(-H_{0}+1\right)-2 \mu^{2} \ln \left(H_{0}\right)+4 D \mu \ln \left(H_{0}\right) \\
& \left.+2 D \hbar v_{F} k-\hbar^{2} v_{F}^{2} k^{2}-2 \hbar v_{F} k \mu\right]\left.\right|_{k}
\end{aligned}
$$


and for semimetal at half-filling it reduces to

$$
\begin{aligned}
F= & -T \lim _{n \rightarrow \infty} \frac{1}{2 \pi 2 \hbar^{2} v_{F}^{2}}\left[-2 \hbar^{2} v_{F}^{2} k^{2} \ln \Gamma\left(-\hbar v_{F} k+n+1\right)+\hbar^{2} v_{F}^{2} k^{2} \ln \left(\frac{\hbar^{2} v_{F}^{2} k^{2}}{T^{2 n+2}}\left(\frac{\Gamma\left(1-\hbar v_{F} k+n\right)}{\Gamma\left(1-\hbar v_{F} k\right)}\right)^{2}\right)\right. \\
& -\hbar^{2} v_{F}^{2} k^{2}-4 \hbar v_{F} k \psi^{(-2)}\left(n-\hbar v_{F} k+1\right)-4 \psi^{(-3)}\left(-\hbar v_{F} k+n+1\right) \\
& \left.+4 \hbar v_{F} k \psi^{(-2)}\left(-\hbar v_{F} k+1\right)+4 \psi^{(-3)}\left(-\hbar v_{F} k+1\right)+k^{2} \ln \Gamma\left(1-\hbar v_{F} k\right)\right]\left.\right|_{k},
\end{aligned}
$$

where $\ln \Gamma$ denotes the logarithm of the Gamma function and $\psi^{(n)}$ is the $n$th derivative of the digamma function. By using the aproximational relation[23]

$$
\ln \left(\frac{\omega^{2}+H_{0}^{2}}{T^{2}}\right) \approx \frac{H_{0}}{T}+2 \ln \left(1+e^{-H_{0} / T}\right)
$$

then the free energy density can be rewritten as

$$
\begin{gathered}
F=-T \frac{1}{2 \pi}\left[\frac{2 T^{2} \operatorname{Li}_{3}\left(-e^{\hbar v_{F} k / T}\right)}{\hbar^{2} v_{F}}+\frac{1}{3} k^{2}\left(\frac{2 \hbar v_{F} k}{T}+3 \ln N_{F}\left(-\hbar v_{F} k\right)-3 \ln N_{F}\left(\hbar v_{F} k\right)\right)\right. \\
\left.-\frac{2 k T \operatorname{Li}_{2}\left(-e^{\hbar v_{F} k / T}\right)}{\hbar v_{F}}\right]\left.\right|_{k},
\end{gathered}
$$

where $\operatorname{Li}_{n}(x)$ is the polylogarithm function. Consider the many-body effect to the 2D Dirac system, the perturbations can be taken into account in the grand-canonical ensemble, where we rewrite the tight-binding model Hamiltonian as

$$
\begin{aligned}
H= & H_{0}+H_{E}+H_{D} \\
= & \sum_{i j}\left(t c_{i}^{\dagger} c_{j}\right)+\sum_{i j} \frac{1}{2} U_{i j} n_{\uparrow} n_{\downarrow} \\
& +V_{0} \sum_{i} c_{i}^{\dagger} c_{i} \delta\left(r-r_{i}\right),
\end{aligned}
$$

where $i j$ is the nearest neighbor sites and $t$ is the nearest neighbor hopping. $n=c_{i}^{\dagger} c_{j} . U_{i j}$ is the Coulomb interaction strength in second term which is the Coulomb exchange interactionrelated term (bilinear). $V_{0}$ is the impurity scattering potential (magnetic or nonmagnetic) in the third term which is the disorder-related term. The creation and annihilate operators are all the particle one here. Then the free energy density (grand potential) is still $F=-T \ln Z$ but with the partion function in interacting case as

$$
\begin{aligned}
Z\left(i \Omega, U_{i j}, V_{0}\right) & =\operatorname{Trexp}\left[\frac{-H+N \mu}{T}\right] \\
& =\int D \psi D \psi^{*} e^{S}
\end{aligned}
$$

where the path integral runs over the Grassman variables, $N$ is the particle number operator, and with the action $S$ reads

$$
S\left(i \Omega, U_{i j}, V_{0}\right)=\sum_{i \Omega} \psi(i \Omega)\left[(i \Omega+\mu) \delta_{i j}-H_{0}-H_{D}\right] \psi^{*}(i \Omega)-\int_{0}^{1 / T} d \tau H_{E}(\tau)
$$

where $\psi(i \Omega)$ is the real Grassmann variable, and the first term is always positive summing over the Fermionic frequency. The perturbaed Green's function which satisfies the Dyson relation 
$G=G_{0}+G \Sigma G_{0}\left(\right.$ or $\left.\Sigma=G_{0}^{-1}-G^{-1}\right)$, can be obtained by the ratio of the partion functions as

$$
\begin{aligned}
G\left(i \Omega, U_{i j}, V_{0}\right) & =\frac{-Z^{\prime}\left(i \Omega, U_{i j}, V_{0}\right)}{Z\left(i \Omega, U_{i j}, V_{0}\right)} \\
& =\frac{-\int D \psi D \psi^{*} e^{S} \psi(i \Omega) \psi^{*}(i \Omega)}{\int D \psi D \psi^{*} e^{S}},
\end{aligned}
$$

The case of $G\left(i \Omega, U_{i j}, V_{0}\right) \neq 1$ clearly indicates broken of the supersymmetry [37]. We consider the $\delta$-type impurity potential in above disorder-term, which indicates the Born approximation. In such case the spin structure is fixed as also been observed in the surface state of the 3D topological insulators, thus the spin rotation is missing which can be observed in the nodalline semimetals, and the spin current operator becomes zero in the helicity basis. The Born approximation guarantees the sign-invariance of the momentum before and after the scattering, and the reversed scattering amplitude has the same value with the origin one 38, In grand canonical ensemble, the spin current vanishes in the thermodynamical limit (and thus with infinite $\mu$ ) due to the vanishing spin density even when beyong the Born approximation. Beyong the $\delta$-type impurity field, the extrinsic spin current emergents and the scattering of both the impurity and the majority particle (with opposite spins) create the Fermionic polaron, and the optical Hall conductivity (in fact this is the only case where the transverse conductivity equals to the Hall conductivity) of the polaron determines the current in direction orthogonal to the external force [39], is related to the current operator by $\sigma_{x y}=J_{x} /\left(-\nabla_{y} V\right)$ in linear response theory where $V$ is the external potential. The current operator here is much smaller than the one in QED just like the group velocity operator which is much smaller than the speed of light. While for the Bosonic polaron for the system immersed in the Bose-Einstein condensates, the interaction is stronger than the Fermionic one due to the higher compresibility of the BEC compared to the Fermionic media. The Bosonic polaron is formed by the Fermionic impurity which dressed by the majority Bosonic excitations.

\section{Optical conductivity}

The destoryed Fermi-liquid behavior can be observed by the singular Bosonic susceptibility at the nesting wavevector, and it can't be found even at low-energy limit (far away from the quantum critical point) when the ultraviolet cutoff applied is infinite during the calculation, as could be found in the many-electron system, for example, when the Fermions coupled to the 1D Ising variable[40] or the fluctuation transverse gauge field [41] or the longitudinal Bosonic excitation [2]. The non-Fermi-liquid phenomenons are widely observed in the heavy-Fermi system and the cuprate materials [42, 43], including the logarithmic divergent specific heat which related to the free energy by $C_{V}=-T \frac{\partial^{2} F}{\partial T^{2}}$. In the presence of monochromatic light, the nondiagonal part of the optical conductivity can be obtained by summing over the eigenvalues:

$$
\begin{aligned}
\sigma_{x y}(\omega) & =i \hbar e^{2} \int \frac{d^{2} k}{(2 \pi)^{2}} \sum_{\varepsilon, \varepsilon^{\prime}} \frac{\left[N_{F}(\varepsilon)-N_{F}\left(\varepsilon^{\prime}\right)\right]\left\langle\varepsilon\left|v_{x}\right| \varepsilon^{\prime}\right\rangle\left\langle\varepsilon^{\prime}\left|v_{y}\right| \varepsilon\right\rangle}{\left(\varepsilon-\varepsilon^{\prime}\right)\left(\varepsilon-\varepsilon^{\prime}+\omega+i 0\right)} \\
= & i \hbar e^{2} \int \frac{d^{2} k}{(2 \pi)^{2}} \sum_{\varepsilon, \varepsilon^{\prime}} \frac{\left[N_{F}(\varepsilon)-N_{F}\left(\varepsilon^{\prime}\right)\right] \frac{i v_{F}^{2}}{4}\left[\left(1-\frac{D}{\varepsilon}\right)\left(1+\frac{D}{\varepsilon^{\prime}}\right)-\left(1-\frac{D}{\varepsilon^{\prime}}\right)\left(1+\frac{D}{\varepsilon}\right)\right]\left(1-\delta_{s s^{\prime}}\right)}{\left(\varepsilon-\varepsilon^{\prime}\right)\left(\varepsilon-\varepsilon^{\prime}+\omega+i 0\right)},
\end{aligned}
$$

where only the retarded Green's function is used in contrast to the Streda one 44]. Here the identify

$$
N_{F}(\varepsilon)\left(1-N_{F}\left(\varepsilon^{\prime}\right)\right)\left(1-e^{\varepsilon / T}\right)=N_{F}(\varepsilon)-N_{F}\left(\varepsilon^{\prime}\right)
$$


is used. The velocity matrix elements are

$$
\begin{aligned}
& \left\langle\varepsilon\left|v_{x}\right| \varepsilon^{\prime}\right\rangle=\frac{v_{F}}{2}\left[s \sqrt{1+\frac{D}{\varepsilon}} \sqrt{1-\frac{D}{\varepsilon^{\prime}}}+s^{\prime} \sqrt{1-\frac{D}{\varepsilon}} \sqrt{1+\frac{D}{\varepsilon^{\prime}}}\right]\left(1-\delta_{s s^{\prime}}\right), \\
& \left\langle\varepsilon^{\prime}\left|v_{y}\right| \varepsilon\right\rangle=\frac{i v_{F}}{2}\left[s^{\prime} \sqrt{1+\frac{D}{\varepsilon^{\prime}}} \sqrt{1-\frac{D}{\varepsilon}}-s \sqrt{1-\frac{D}{\varepsilon}} \sqrt{1+\frac{D}{\varepsilon^{\prime}}}\right]\left(1-\delta_{s s^{\prime}}\right),
\end{aligned}
$$

where the electron/hole indices have $s s^{\prime}=-1$ during the optical transition due to the Pauli exclusion principle, while the spin indices $\sigma_{z}$ before and after transition are invariant when (both the intrinsic and extrinsic) Rashba-coupling are negligible, otherwise the spin index changes since it's nomore the good quantum number. In case that the Fermi level lies within the band gap, the diagonal elements of the conductivity are zero (and thus implies the $C_{4}$ symmetry of the system since $\sigma_{x x}=\sigma_{y y}$ ), while the non-diagonal elements becomes independent of the Dirac-mass due to the vanishing classical term[44. Note that the result of $\sigma_{x x}=\sigma_{y y}$ as well as the $\sigma_{x y}=-\sigma_{y x}$ also appear in the optical limit with $q \rightarrow 0$ (also called the local limit) Taking into account the effect of chemical potential, then the Hall conductivity is composed of two parts:

$$
\begin{aligned}
\sigma_{x y}(\omega)= & i \hbar e^{2} \int \frac{d^{2} k}{(2 \pi)^{2}} \Theta(\Lambda-\varepsilon)\left[\frac{\left\langle\varepsilon\left|v_{x}\right| \varepsilon^{\prime}\right\rangle\left\langle\varepsilon^{\prime}\left|v_{y}\right| \varepsilon\right\rangle}{\left(\varepsilon-\varepsilon^{\prime}\right)\left(\varepsilon-\varepsilon^{\prime}+\omega+i 0\right)}+\frac{\left\langle\varepsilon^{\prime}\left|v_{x}\right| \varepsilon\right\rangle\left\langle\varepsilon\left|v_{y}\right| \varepsilon^{\prime}\right\rangle}{\left(\varepsilon-\varepsilon^{\prime}\right)\left(-\varepsilon+\varepsilon^{\prime}+\omega+i 0\right)}\right], \\
\sigma_{x y}(\omega, \mu)= & i \hbar e^{2} \int \frac{d^{2} k}{(2 \pi)^{2}} \Theta(\mu-\varepsilon)\left\{\left[\frac{\left\langle\varepsilon\left|v_{x}\right| \varepsilon\right\rangle\left\langle\varepsilon\left|v_{y}\right| \varepsilon\right\rangle}{\left(\varepsilon+\varepsilon^{\prime}\right)\left(-\varepsilon-\varepsilon^{\prime}+\omega+i 0\right)}+\frac{\left\langle\varepsilon\left|v_{x}\right| \varepsilon\right\rangle\left\langle\varepsilon\left|v_{y}\right| \varepsilon\right\rangle}{\left(\varepsilon+\varepsilon^{\prime}\right)\left(\varepsilon+\varepsilon^{\prime}+\omega+i 0\right)}\right]\right. \\
& \left.-\left[\frac{\left\langle\varepsilon\left|v_{x}\right| \varepsilon^{\prime}\right\rangle\left\langle\varepsilon^{\prime}\left|v_{y}\right| \varepsilon\right\rangle}{\left(\varepsilon-\varepsilon^{\prime}\right)\left(\varepsilon-\varepsilon^{\prime}+\omega+i 0\right)}+\frac{\left\langle\varepsilon^{\prime}\left|v_{x}\right| \varepsilon\right\rangle\left\langle\varepsilon\left|v_{y}\right| \varepsilon^{\prime}\right\rangle}{\left(\varepsilon-\varepsilon^{\prime}\right)\left(-\varepsilon+\varepsilon^{\prime}+\omega+i 0\right)}\right]\right\},
\end{aligned}
$$

where the first part corresponds to the case that the chemical potential is small than the Diracmass while the second part is opposites. While the longitidinal optical conductivity reads

$$
\sigma_{x x}(\omega)=\sigma_{x x}^{\text {inter }}(\omega)=-e^{2} \pi \hbar \int \frac{d^{2} k}{(2 \pi)^{2}} \frac{\left|\left\langle\varepsilon\left|v_{x}\right| \varepsilon^{\prime}\right\rangle\right|^{2}}{\varepsilon-\varepsilon^{\prime}} \delta\left(\omega+i 0+\varepsilon-\varepsilon^{\prime}\right),
$$

where the intraband part vanishes unless at finite temperature and at infrared limit (nearly zero photon energy).

Different to the hopping-current-related conductivity, the dissipation-current-related conductivity remains finite in static limit and proportional to $i / \omega+\pi \delta(\omega)$ [36] where $\delta$ is the Dirac- $\delta$ function here, but this part of the conductivity is negligible when under a magnetic field or at low-temperature. For the case of large band gap, the frequency about the optical tansition during the intraband process is much larger than the interband one as shown in the WSe 2 [45].

\section{Observable quantities in Dirac/Weyl systems with higher-order dispersion}

\subsection{D Dirac system}

Next we discuss the 2D topological insulator (TI) with higher-order dispersion and small momentum-dependent mass term, whose Hamiltonian reads

$$
H_{0}^{m}=\frac{k_{0}^{2-m} a}{2} \hbar v_{F}|\mathbf{k}|^{m}(\hat{k} \cdot \sigma)+\left(c_{1} \sigma_{z}+c_{2}|\mathbf{k}|^{m} \sigma_{z} \tau_{z}\right)-\mu
$$

where we assume the Dirac-mass is momentum-dependent and controlled by the materialrelated constant $c_{1}$ and $c_{2}$, the $k_{0}$ is another material-related constant in unit of momentum[21] 
and $a$ is the lattice spacing[46], e.g., it equals to $\sqrt{3} / 2$ times of the lattice constant in the graphene-like hexagonal lattice system. In the following we denote $\xi=\frac{k_{0}^{2-m} a}{2} \hbar v_{F}$. The term $(\hat{k} \cdot \sigma)$ here only appears in the chiral systems with spin-momentum locking, while for the nonchiral systems, it's usually replaced by the spin operator $\sigma_{z}$ and the interband transition also vanishes in such case. The (in-plane) momentum-dependent mass term $\left(c_{1}+c_{2}|\mathbf{k}|^{m}\right)$ here is similar to the effect of next-nearest-neighbor (intrinsic) Rashba coupling. Here we note that, we try to present a discussion for in the 2D Dirac system extend to the generic order $m$ which also related to the Chern number in gapless case, and it's not just applicable to the 2D TI, but also to the multilayer TI which with a single 2D Dirac node per surface Brillouin zone 47, 48]. The order $m$ controls the in-plane band dispersion, for example, $m=1$ for the (topologically protected; which not exists currently in 3D real space[49]) linear Dirac dispersion, $m=2$ for the quadratic dispersion, $m=3,4$ for the trigonal warping system as found in the monolayer $\mathrm{MoS}_{2}$ [50]. The above low-energy effective Hamiltonian of the high-order topological Dirac system can be rewritten in the tight-binding approximation as

$$
H_{0}^{m}=\int \frac{d^{2} k}{(2 \pi)^{2}} \psi_{k \sigma_{z}}^{\dagger} H_{k} \psi_{k \sigma_{z}}-\mu
$$

where $\psi_{k \sigma}^{\dagger}=\left(c_{k \sigma_{z}}^{\dagger} d_{k \sigma_{z}}^{\dagger}\right)$ is the spinor field with two creation operators correspond to the two sublattices degrees of freedom, and

$$
H_{k}=\xi\left(\begin{array}{cccc}
c_{1}+c_{2}|\mathbf{k}|^{m} & \left(k_{x}-i k_{y}\right)^{m} & 0 & 0 \\
\left(k_{x}+i k_{y}\right)^{m} & c_{1}-c_{2}|\mathbf{k}|^{m} & 0 & 0 \\
0 & 0 & c_{1}-c_{2}|\mathbf{k}|^{m} & \left(k_{x}-i k_{y}\right)^{m} \\
0 & 0 & \left(k_{x}+i k_{y}\right)^{m} & c_{1}+c_{2}|\mathbf{k}|^{m}
\end{array}\right) .
$$

In noninteracting case, the Bosonic propagator is overdamped with the gapless longitudinal excitations (or order parameter fluctuation) by the Landau damping, it's also found that the Landau damping of the multi-Weyl semimetal is weaker than that of the marginal Fermi liquid[30] which is distinct from the normal non-Fermi-liquid states. The dispersion of the multi-node Dirac system can be obtained by solving above Hamiltonian as

$$
\begin{aligned}
\varepsilon^{m} & = \pm \sqrt{c_{1}^{2}+2 c_{1} c_{2} k^{m}+c_{2}^{2} k^{2 m}+\mu^{2}+k^{2 m} \xi^{2}-2 \eta|\mathbf{k}|^{m} \mu \xi \cos \theta} \\
& \approx \pm \sqrt{c_{1}^{2}+2 c_{1} c_{2} k^{m}+c_{2}^{2}|\mathbf{k}|^{2 m}+|\mathbf{k}|^{2 m} \xi^{2}} \\
& = \pm \sqrt{\left(c_{1}+c_{2}|\mathbf{k}|^{m}\right)^{2}+|\mathbf{k}|^{2 m} \xi^{2}}
\end{aligned}
$$

where we rewrite the term $\frac{k_{0}^{2-m} a}{2} \hbar v_{F}$ as the scale-dependent parameter $\xi$.

For the above multi-node dispersion, the eigenvectors for conduction band and valence band are

$$
\left|\Psi_{k}^{\lambda}\right\rangle=\frac{e^{i \mathbf{k} \cdot \mathbf{r}}}{\sqrt{S}} \frac{1}{\sqrt{2}}\left(\begin{array}{c}
\cos \frac{\alpha}{2} \\
-\lambda \sin \frac{\alpha}{2} e^{\lambda i m \theta}
\end{array}\right),
$$

where $\alpha=\arctan \frac{\xi|\mathbf{k}|^{m}}{\left(c_{1}+c_{2}|\mathbf{k}|^{m}\right)}$ and $\theta=\arctan \frac{k_{y}}{k_{x}}$ is the polar angle of $\mathbf{k} . \lambda= \pm 1$ correspond to the electron and hole states, respectively. Thus for electron and hole states, we have the following spinor parts

$$
\begin{aligned}
\left|\Psi_{k}^{+}\right\rangle_{s} & =\frac{1}{\sqrt{2}}\left(\begin{array}{c}
\cos \frac{\alpha}{2} \\
-\sin \frac{\alpha}{2} e^{i m \theta}
\end{array}\right), \\
\left|\Psi_{k}^{-}\right\rangle_{s} & =\frac{1}{\sqrt{2}}\left(\begin{array}{c}
\sin \frac{\alpha}{2} \\
\cos \frac{\alpha}{2} e^{i m \theta}
\end{array}\right) .
\end{aligned}
$$


Then the overlap factor (the form factor within the electronic susceptibility) for this chiral model reads

$$
\begin{aligned}
\left|\left\langle\Psi_{k+q}^{\lambda^{\prime}} \mid \Psi_{k}^{\lambda}\right\rangle\right|^{2} & =\left(\frac{e^{-i \mathbf{k}^{\prime} \cdot \mathbf{r}+i \mathbf{k} \cdot \mathbf{r}}}{2 S}\right)^{2}\left[\cos \frac{\alpha}{2} \cos \frac{\alpha^{\prime}}{2}+\lambda \lambda^{\prime} \sin \frac{\alpha}{2} \sin \frac{\alpha^{\prime}}{2} e^{\lambda i m\left(\theta-\theta^{\prime}\right)}\right]^{2} \\
& =\frac{e^{2 i\left(\mathbf{k} \cdot \mathbf{r}-\mathbf{k}^{\prime} \cdot \mathbf{r}\right)}}{4 S^{2}}\left[\cos \left(\frac{\alpha}{2}-\frac{\alpha^{\prime}}{2}\right)\left(-1-\lambda \lambda^{\prime} e^{i \lambda m\left(\theta-\theta^{\prime}\right)}\right)+\cos \left(\frac{\alpha}{2}+\frac{\alpha^{\prime}}{2}\right)\left(-1+\lambda \lambda^{\prime} e^{i \lambda m\left(\theta-\theta^{\prime}\right)}\right)\right]^{2},
\end{aligned}
$$

where $\mathbf{k}^{\prime}=\mathbf{k}+\mathbf{q}, \theta^{\prime}=\arctan \frac{k_{y}^{\prime}}{k_{x}^{\prime}}$, and $\alpha^{\prime}=\arctan \frac{\xi\left|\mathbf{k}^{\prime}\right|^{m}}{\left(c_{1}+c_{2}\left|\mathbf{k}^{\prime}\right|^{m}\right)}$. The form factor here ensuring the electronic susceptibility only contributed by the overlap of electron-hole pair (i.e., the scattering between conduction band and valence band). It's obvious that we do not consider the effect of spin state here, which means that we only consider the density-density correlation which is in longitudinal channel. In another case, if the spin or pseudospin degrees of freedom are also been traced over, the current tensors should be discussed in diagonal and non-diagonal cases [17, 51, 52. However, if we restrict on the longitudinal channel with weak short-range interaction (in non-Fermi liquid picture), we found that no matter for the graphene-like Dirac systems or the helical liquid systems (with eigenvectors $\frac{e^{i \mathbf{k} \cdot \mathbf{r}}}{\sqrt{S}} \frac{1}{\sqrt{2}}\left(\cos \frac{\alpha}{2},-i \lambda \sin \frac{\alpha}{2} e^{i \lambda m \theta}\right)^{T}$ ), which couples with the momentum by the pseudospin and spin degrees of freedom respectively, the overlap function is the same (i.e., Eq.(40)), while for the massless case, their overlap function is $\cos ^{2}\left(\frac{m \theta^{\prime}-m \theta}{2}\right)$.

At half-filling, the Fermi level crosses the multi-band touching point and the Coulomb interaction remains long-ranged as described by the Bosonic field, due to the poor screening to the electron-electron Coulomb interaction. The imaginary part of the exchange-induced self-energy is related to the quasiparticle relaxation time (lifetime), while the real part is related to the interaction strength and the quaisparticle weight. Beyond the instantaneous approximation induced by the scalar potential, the exchange-induced self-energy containing Bosonic frequency reads (see Appendix.A for a further calculation and discussion)

$$
\Sigma^{m}(\omega, k)=-g^{2} \int \frac{d \Omega}{2 \pi} \frac{d^{2} k}{(2 \pi)^{2}} G_{0}\left(\omega+\Omega, k^{\prime}\right) \frac{1}{q-\Sigma_{b}(\Omega, q)},
$$

where the last term of the above expression is the dressed Coulomb potential. $\Sigma_{b}^{m}(\Omega, q)$ is the Bosonic self-energy (i.e., the dynamical polarization here) which reads

$$
\Sigma_{b}^{m}(\Omega, q)=g_{s} g_{v} g^{2} \int \frac{d \Omega}{2 \pi} \frac{d^{2} k}{(2 \pi)^{2}} \operatorname{Tr}\left[\sigma_{0} G_{0}^{m}\left(\Omega^{\prime}, k^{\prime}\right) \sigma_{0} G_{0}^{m}(\Omega, k)\right] .
$$

then the multi-Dirac-node bare Green's function reads

$$
\begin{aligned}
G_{0}^{m}(\Omega, k) & =\frac{\Omega+i 0+H_{0}^{m}}{(\Omega+i 0)^{2}-H_{0}^{m 2}} \\
& =\frac{\Omega+i 0+\xi k^{m}(\hat{k} \cdot \sigma)+\left(c_{1}+c_{2} k^{m}\right) \sigma_{z} \tau_{z}-\mu}{(\Omega+i 0)^{2}-\left(\xi k^{m}(\hat{k} \cdot \sigma)+\left(c_{1}+c_{2} k^{m}\right) \sigma_{z} \tau_{z}-\mu\right)^{2}} .
\end{aligned}
$$

Base on this Green's function, the dynamical polarization is available by the above expression but it's too verbose to express which contains a hypergeometric function whose parameters are all related to the order $m$. We then turn to a more concise expression at zero-temperature limit 
which reads

$$
\begin{aligned}
\Sigma_{b}^{m}(\Omega, q) & =\int \frac{d^{2} k}{(2 \pi)^{2}} \sum_{s s^{\prime}} \frac{g_{s} g_{v}}{2} \frac{1+s s^{\prime} \cos b}{\Omega+i 0+s \varepsilon^{m}-s^{\prime} \varepsilon^{m^{\prime}}}, \\
\cos b & =\frac{k^{m}+q \cos a}{\sqrt{k^{2}+q^{2}+2 k^{m} q \cos a}}=1-\frac{q^{2} \sin ^{2} a}{2 k^{2 m}}+O\left(q^{3}\right), \\
\varepsilon^{m}-\varepsilon^{m^{\prime}} & =-\frac{m q k^{m-1}\left(c_{1} c_{2}+c_{2}^{2} k^{m}+\xi^{2} k^{m}\right)}{\sqrt{c_{1}^{2}+2 c_{1} c_{2} k^{m}+c_{2}^{2} k^{2 m}+\xi^{2} k^{2 m}}}+O\left(q^{2}\right),
\end{aligned}
$$

where $b$ is the angle between $k$ and $k^{\prime}$ and $a$ is the angle between $k$ and $q$. The dynamical polarization can be devided into the intraband and interband parts:

$$
\begin{aligned}
\Sigma_{b}^{m}(\Omega, q) & =\Sigma_{b}^{m, \text { intra }}(\Omega, q)+\Sigma_{b}^{m, \text { inter }}(\Omega, q), \\
\Sigma_{b}^{m, \text { intra }}(\Omega, q) & =\frac{g_{s} g_{v}}{2} \int_{0}^{2 \pi} d \theta \int_{0}^{k_{F}} k d k\left(\frac{1}{\Omega+i 0+\varepsilon^{m}-\varepsilon^{m^{\prime}}}-\frac{1}{\Omega+i 0-\varepsilon^{m}+\varepsilon^{m^{\prime}}}\right)(1+\cos b) \\
= & \Sigma_{b 1}^{m, \text { intra }}(\Omega, q)+\Sigma_{b 2}^{m, \text { intra }}(\Omega, q), \\
\Sigma_{b}^{m, \text { inter }}(\Omega, q) & =\frac{g_{s} g_{v}}{2} \int_{0}^{2 \pi} d \theta \int_{k_{F}}^{\Lambda} k d k\left(\frac{1}{\Omega+i 0+\varepsilon^{m}+\varepsilon^{m^{\prime}}}-\frac{1}{\Omega+i 0-\varepsilon^{m}-\varepsilon^{m^{\prime}}}\right)(1-\cos b) \\
= & \Sigma_{b 1}^{m, \text { inter }}(\Omega, q)+\Sigma_{b 2}^{m, \text { inter }}(\Omega, q),
\end{aligned}
$$

where the first term of the intraband part can be obtained after some algebra as

$$
\begin{aligned}
& \Sigma_{b 1}^{m, i n t r a}(\Omega, q)=k^{1-m} \sqrt{c_{1}^{2}+2 c_{1} c_{2} k^{m}+\left(c_{2}^{2}+\xi^{2}\right) k^{2 m}} \\
& {\left[4 k^{2}(m-1) F_{1}\left(\frac{3}{m}-1 ;-\frac{1}{2},-\frac{1}{2} ; \frac{3}{m} ;-\frac{k^{m}\left(c_{2}^{2}+\xi^{2}\right)}{c_{1} c_{2}+\sqrt{-c_{1}^{2} \xi^{2}}}, \frac{k^{m}\left(c_{2}^{2}+\xi^{2}\right)}{-c_{1} c_{2}+\sqrt{-c_{1}^{2} \xi^{2}}}\right)\right.} \\
& \left.-(m-3) q^{2} \sin ^{2} a F_{1}\left(\frac{1}{m}-1 ;-\frac{1}{2},-\frac{1}{2} ; \frac{1}{m} ;-\frac{k^{m}\left(c_{2}^{2}+\xi^{2}\right)}{c_{1} c_{2}+\sqrt{-c_{1}^{2} \xi^{2}}}, \frac{k^{m}\left(c_{2}^{2}+\xi^{2}\right)}{-c_{1} c_{2}+\sqrt{-c_{1}^{2} \xi^{2}}}\right)\right] \\
& \left.\frac{1}{2 c_{1} c_{2}(m-3)(m-1) m q \sqrt{\frac{-\sqrt{-c_{1}^{2} \xi^{2}}+c_{1} c_{2}+c_{2} k^{m}+\xi^{2} k^{m}}{c_{1} c_{2}-\sqrt{-c_{1}^{2} \xi^{2}}}} \sqrt{\frac{\sqrt{-c_{1}^{2} \xi^{2}}+c_{1} c_{2}+c_{2} k^{m}+\xi^{2} k^{m}}{c_{1} c_{2}+\sqrt{-c_{1}^{2} \xi^{2}}}}}\right|_{0} ^{k_{F}},
\end{aligned}
$$

where $F_{1}\left(a ; b, b^{\prime} ; c ; d, d^{\prime}\right)=\sum_{m, n=0}^{\infty} \frac{(a)_{m+n}(b)_{m}\left(b^{\prime}\right)_{n}}{m ! n !(c)_{m+n}} d^{m} d^{\prime n}$ is the Appell hypergeometric function. The other three terms can be obtained through the same way, and then the exchange-induced self-energy can also be obtained. Note that for the non-chiral Fermions, like the ones in 2D electron gas, $\Sigma_{b}^{m, \text { inter }}(\Omega, q)$ vanishes since $\cos b=1$.

\subsection{D Dirac system}

While for the 3D Dirac semimetals [53, 54, 55] like the $\mathrm{Na}_{3} \mathrm{Bi}$ or $\mathrm{Cd}_{3} \mathrm{As}_{2}, \mathrm{PtTe}_{2}$, the the chiral anomaly emerges since in odd space dimensions the anti-commute relation about the $\gamma_{5}$ matrix is allowed, and each Dirac node resolved into two Weyl nodes 54 arrange along the $z$-direction of the momentum space and with opposite chirality. The Hamiltonian of the simplest 3D Dirac and Weyl semimetal are $H=\sum_{i} \hbar v_{i} k_{i} \sigma_{i}(i=x, y, z)$ and $H=\chi \sum_{i} \hbar v_{i} k_{i} \sigma_{i}+\chi \hbar v_{z}\left(k_{z}-\chi \delta k_{z}\right)$ $(i=x, y)$, respectively. The chiral effect gives the signs \pm to $\xi$. In 3D Dirac/Weyl semimetal, the perturbations can remove the nodal line and leave the nodes[56], while the nodes can not be removed but can only be shifted 47]. As we mentioned above, since the spin rotation is missing due to the Dirac $\delta$-type impurity field, the rotational invariance is presented, which is 
also partly due to the disorder averaging [57, 58], and thus the disorder-induced self-energy is independent of the external momentum, which reads

$$
\Sigma^{D}(\omega)=\frac{n_{i} V_{0}^{2}}{\hbar^{2}} \int \frac{d^{3} k}{(2 \pi)^{3}} \Gamma_{0} G_{0}^{m T}(\omega, k) \Gamma_{0}
$$

where $\Gamma_{0}$ is the irreducible vertex function which doesn't contains the Levi-Civita symbol here unlike the one in Ref.[71]. The vertex correction vanishes when it contains only the exchangeinduced self-energy correction in instantaneous approximation, which can be obtained by the Ward identity $\frac{\partial \Sigma(\Omega, k)}{\partial \omega}=\Gamma(\Omega, k)$, besides, the vertex correction also vanishes in the large speciescase (large $g$ ) or when the integration momentum shell vanishes (the $\mathrm{RG}$ flow parameter $\ell=1$ ). $n_{i}$ here is the impurity concentration, $V_{0}$ is the impurity scattering potential (a scalar potential when only with the nonmagnetic impurity and without the magnetic impurity).

For the 3D Dirac/Weyl system, we can write the Hamiltonian as

$$
H_{0}^{m T}=\frac{k_{0}^{2-m} a}{2} \hbar v_{F}|\mathbf{k}|^{m}(\hat{k} \cdot \sigma)+\hbar v_{z} k_{z} \sigma_{3}+\left(c_{1}+c_{2}|\mathbf{k}|^{m} \sigma_{z} \tau_{z}\right)-\mu
$$

the energy can be obtained as

$$
\begin{aligned}
\varepsilon^{m T}= & -\mu+c_{1}+c_{2}|\mathbf{k}|^{m} \mp \\
& \sqrt{\left(\mu-\left(c_{1}+c_{2}|\mathbf{k}|^{m}\right)\right)^{2}+\hbar^{2} v_{z}^{2} k_{z}^{2}+|\mathbf{k}|^{2 m} \xi^{2}+2|\mathbf{k}|^{m} \xi \cos \theta\left(-\mu+\left(c_{1}+c_{2}|\mathbf{k}|^{m}\right)\right)}, \\
\approx & -\mu+c_{1}+c_{2}|\mathbf{k}|^{m} \mp \sqrt{\hbar^{2} v_{z}^{2} k_{z}^{2}+|\mathbf{k}|^{2 m} \xi^{2}},
\end{aligned}
$$

and the eigenvectors can still be written in a form similar to Eq.(38), but the factor $\alpha$ should be replaced by $\alpha^{T}=\arctan \frac{\xi|\mathbf{k}|^{m}}{D^{T}}$ where $D^{T}$ is the gap opened by the collective effect of in-plane mass term $\left(c_{1}+c_{2}|\mathbf{k}|^{m}\right)$ and the $z$-direction momentum. Note that for the case of $\mu \neq 0$, the gap produced by the chemical potential should also be taken into account, which should as large as $2|\mu|$. And for the ultrathin film of Weyl semimetal, since the two Weyl nodes with opposite chirality are very close, and the $k_{z}$ is quantized [22, 75], which gives rise to a mass.

The velocity operators can be obtained by using the relation $v_{\alpha}=\frac{\partial H_{0}^{m}}{\hbar \partial k_{\alpha}}(\alpha=x, y, z)$ :

$$
\begin{aligned}
v_{x} & =\frac{1}{\hbar}\left[\left(\begin{array}{cc}
m \xi|\mathbf{k}|^{m-1} e^{-i(m-1) \theta} & 0 \\
0 & m \xi|\mathbf{k}|^{m-1} e^{i(m-1) \theta}
\end{array}\right) \sigma_{x}+m c_{2}|\mathbf{k}|^{m-1} \cos \theta \sigma_{3}\right] \\
& =\frac{1}{\hbar}\left(\begin{array}{cc}
m c_{2}|\mathbf{k}|^{m-1} \cos \theta & m \xi|\mathbf{k}|^{m-1} e^{-i(m-1) \theta} \\
m \xi|\mathbf{k}|^{m-1} e^{i(m-1) \theta} & -m c_{2}|\mathbf{k}|^{m-1} \cos \theta
\end{array}\right), \\
v_{y} & =\frac{1}{\hbar}\left[\left(\begin{array}{cc}
m \xi|\mathbf{k}|^{m-1} e^{-i(m-1) \theta} & 0 \\
0 & m \xi|\mathbf{k}|^{m-1} e^{i(m-1) \theta}
\end{array}\right) \sigma_{y}+m c_{2}|\mathbf{k}|^{m-1} \sin \theta \sigma_{3}\right] \\
& =\frac{1}{\hbar}\left(\begin{array}{cc}
m c_{2}|\mathbf{k}|^{m-1} \sin \theta & -i m \xi|\mathbf{k}|^{m-1} e^{-i(m-1) \theta} \\
i m \xi|\mathbf{k}|^{m-1} e^{i(m-1) \theta} & -m c_{2}|\mathbf{k}|^{m-1} \sin \theta
\end{array}\right),
\end{aligned}
$$

where the relation $\frac{\partial k}{\partial k_{\alpha}}=\frac{k_{\alpha}}{k}(\alpha=x, y, z)$ is used. Here we write the third Pauli matrix as $\sigma_{3}$ to distinguish from the spin operator in $z$ direction. The velocity matrix elements can be obtained base on the above velocity operators,

$$
\begin{aligned}
& \left\langle\varepsilon\left|v_{x}\right| \varepsilon^{\prime}\right\rangle=\mp \frac{|\mathbf{k}|^{m-1} m \sqrt{c_{2}^{2}+2 \xi^{2}+c_{2}^{2} \cos (2 \theta)}}{\sqrt{2}}\left(1-\delta_{s, s^{\prime}}\right), \\
& \left\langle\varepsilon\left|v_{y}\right| \varepsilon^{\prime}\right\rangle=\mp \frac{i|\mathbf{k}|^{m-1} m \sqrt{-c_{2}^{2}-2 \xi^{2}+c_{2}^{2} \cos (2 \theta)}}{\sqrt{2}}\left(1-\delta_{s, s^{\prime}}\right) .
\end{aligned}
$$


Considering only the spin-flipping during the scattering, the optical conductivity can be obtained as $\sigma(\omega)=\sigma_{i j}+\sigma_{i j}^{\prime}$, with

$$
\sigma_{i j}=-i \hbar e^{2} \int \frac{d^{3} k}{(2 \pi)^{3}} \frac{N_{F}\left(\varepsilon_{-k}\right)-N_{F}\left(\varepsilon_{+k}\right)}{\varepsilon_{-k}-\varepsilon_{+k}} \frac{\left\langle\varepsilon^{\prime}\left|v_{i}\right| \varepsilon\right\rangle\left\langle\varepsilon\left|v_{j}\right| \varepsilon^{\prime}\right\rangle}{\hbar \omega-\varepsilon_{+k}+\varepsilon_{-k}+i \eta}
$$

for scattering from up-spin to down-spin, and

$$
\sigma_{i j}^{\prime}=-i \hbar e^{2} \int \frac{d^{3} k}{(2 \pi)^{3}} \frac{N_{F}\left(\varepsilon_{+k}\right)-N_{F}\left(\varepsilon_{-k}\right)}{\varepsilon_{+k}-\varepsilon_{-k}} \frac{\left\langle\varepsilon\left|v_{i}\right| \varepsilon^{\prime}\right\rangle\left\langle\varepsilon^{\prime}\left|v_{j}\right| \varepsilon\right\rangle}{\hbar \omega-\varepsilon_{-k}+\varepsilon_{+k}+i \eta},
$$

for scattering from down-spin to up-spin. Then we can obtain

$$
\begin{aligned}
& \sigma_{x y}=\frac{-i \hbar e^{2}}{(2 \pi)^{3}} \frac{1.5708 i k m^{2}}{c_{2}^{2}(i \eta+\hbar \omega)} \sqrt{c_{2}^{2}+2 \xi^{2}+c_{2}^{2} \cos (2 \theta)} \sqrt{-c_{2}^{2}-2 \xi^{2}+c_{2}^{2} \cos (2 \theta)} \\
& {\left[-i \eta-\hbar \omega+2 c_{12} F_{1}\left[1, \frac{1}{m} ; 1+\frac{1}{m} ;-\frac{c_{2} k^{m}}{c_{1}}\right]\right.} \\
& +\left.\left(i \eta-2 c_{1}+\hbar \omega\right)_{2} F_{1}\left[1, \frac{1}{m} ; 1+\frac{1}{m} ;-\frac{c_{2} k^{m}}{-5 i \eta+c_{1}-0.5 \hbar \omega}\right]\right|_{k}, \\
& \sigma_{x x}=\frac{-i \hbar e^{2}}{(2 \pi)^{3}} \frac{1.5708 k m^{2}}{c_{2}^{2}(i \eta+\hbar \omega)}\left(c_{2}^{2}+2 \xi^{2}+c_{2}^{2} \cos (2 \theta)\right) \\
& {\left[-i \eta-\hbar \omega+2 c_{12} F_{1}\left[1, \frac{1}{m} ; 1+\frac{1}{m} ;-\frac{c_{2} k^{m}}{c_{1}}\right]\right.} \\
& +\left.\left(i \eta-2 c_{1}+\hbar \omega\right)_{2} F_{1}\left[1, \frac{1}{m} ; 1+\frac{1}{m} ;-\frac{c_{2} k^{m}}{-5 i \eta+c_{1}-0.5 \hbar \omega}\right]\right|_{k}, \\
& \sigma_{y y}=\frac{-i \hbar e^{2}}{(2 \pi)^{3}} \frac{-1.5708 k m^{2}}{c_{2}^{2}(i \eta+\hbar \omega)}\left(-c_{2}^{2}-2 \xi^{2}+c_{2}^{2} \cos (2 \theta)\right) \\
& {\left[-i \eta-\hbar \omega+2 c_{12} F_{1}\left[1, \frac{1}{m} ; 1+\frac{1}{m} ;-\frac{c_{2} k^{m}}{c_{1}}\right]\right.} \\
& +\left.\left(i \eta-2 c_{1}+\hbar \omega\right)_{2} F_{1}\left[1, \frac{1}{m} ; 1+\frac{1}{m} ;-\frac{c_{2} k^{m}}{-5 i \eta+c_{1}-0.5 \hbar \omega}\right]\right|_{k} .
\end{aligned}
$$

Similarly, the $\sigma_{i j}^{\prime}$ can be obtained in the same way. The results of the optical conductivity are presented in the Fig.1-3, where we can see that for optical transition from up-spin to downspin (Fig.1), or the total one (Fig.3), the longitudinal and transverse conductivities are linear with frequency $\omega$ in the low-energy region, which is consistent with the result of Ref.[21]. In Fig.1, the slope of such linear conductivity can be approximately written as $10 \mathrm{~m} \frac{i \hbar e^{2}}{(2 \pi)^{3}}$, while that of the total optical conductivity is independent of $m$. From Fig.1, we can also see that the optical conductivity increases with photon frequency and then saturates in large value of $\omega$. For larger $m$, the saturation happen earlier. The effect of angle $\theta$ (i.e., the degree of anisotropy) is enhanced with the increase of $m$. But from Fig.3, we can see that for transverse total optical conductivity, the effect of anisotropy is supressed. The total optical conductivity is proportional to the order $m$. We also find that, for isotropic case $(\theta=\Phi=\pi / 4), \sigma_{x x}=\sigma_{y y}$, which implies that the system has 4 -fold rotational symmetry $\left(C_{4}\right.$ symmetry) in isotropic case no matter how large the $m$ is.

\subsection{D Weyl system}

The Dirac nodes can be divided into the Weyl nodes along the $z$-direction by using the projection operator[71], and the Hamiltonian with topological winding number $\chi$ (chirality) 
reads

$$
H_{0}^{m T}=\frac{k_{0}^{2-m} a}{2} \hbar v_{F} k^{m}(\hat{k} \cdot \sigma)+\chi \hbar v_{z}\left(k_{z}-\chi \delta k_{z}\right) \sigma_{z}+\left(c_{3}+c_{4}\left(k_{z}-\chi \delta k_{z}\right)^{n}\right) \sigma_{z} \tau_{z}-\mu_{\chi},
$$

where $\mu_{\chi}$ is the chemical potential with chirality $\chi= \pm 1$ and $\mu_{+}=\mu_{-}=\mu$ in undoped case. $\delta k_{z}$ is the distance in momentum space removed from the previous Dirac node which explicitly breaks the time-reversal symmetry. $v_{z}=\frac{a t_{\perp} \sin \left(\delta k_{z} a\right)}{\hbar}$ is the $z$-direction velocity. Here we define $k_{x}=k \cos \theta, k_{y}=k \sin \theta, k=\mathbf{k} \sin \varphi, k_{z}=\mathbf{k} \cos \varphi$, and still use the defination $\frac{k_{0}^{2-m} a}{2} \hbar v_{F}=\xi$. The first term of the above Hamiltonian contains no out-of-plane components, which indicates the untilted type-I Weyl semimetal when the mass term is missing. The mass term is dominated by the momentum $k_{z}$ here rather than the in-plane momentum as shown in the previous model and it explicitly breaks the inversion symmetry. Then eigenvalues can be obtained as

$$
\begin{aligned}
\varepsilon^{m T}= & -\mu-\hbar v_{z}\left(\delta k_{z}-\chi k_{z}\right) \\
& \mp \sqrt{\left[c_{3}+c_{4}\left(k_{z}-\chi \delta k_{z}\right)^{n}\right]^{2}+k^{2 m} \xi^{2}+\left(\delta k_{z}-k_{z} \chi\right)^{2}-2 \eta k^{m} \xi\left(\mu+\hbar v_{z}\left(\delta k_{z}-k_{z} \chi\right)\right) \cos \theta} \\
\approx & -\mu-\hbar v_{z}\left(\delta k_{z}-\chi k_{z}\right) \mp \sqrt{\left[c_{3}+c_{4}\left(k_{z}-\chi \delta k_{z}\right)^{n}\right]^{2}+k^{2 m} \xi^{2}} .
\end{aligned}
$$

The imaginary part and real part of the bare Green's function (Fermion propagator) $G_{0}^{m T}(\omega, k)$ are

$$
\begin{aligned}
\operatorname{Im} G_{0}^{m T}(\omega, k) & =-\pi \delta\left(\omega-\varepsilon^{m T}\right) \\
\operatorname{Re} G_{0}^{m T}(i \omega, k) & =\frac{2}{\pi} \int_{0}^{\infty} d \omega \frac{\omega}{\omega^{2}-(i \omega)^{2}} \operatorname{Im} G_{0}^{m T}(\omega, k) \\
& =\frac{-2 \varepsilon^{m T} \theta\left(\varepsilon^{m T}\right)}{\left(\varepsilon^{m T}\right)^{2}+\omega^{2}}
\end{aligned}
$$

where the Sokhotski-Plemelj theorem and Kramers-Kronig relation are used. $\theta(x)$ here is the step function.

Using the eigenvalue

$$
\varepsilon^{m T}=-\mu-\hbar v_{z}\left(\delta k_{z}-\chi(k \cos \varphi)\right) \mp \sqrt{c_{3}^{2}+(k \sin \varphi)^{2 m} \xi^{2}},
$$

where we further set $c_{4}=0$, then after some algebra, the above disorder-induced self-energy can be obtained as

$$
\begin{aligned}
\Sigma^{D}(\omega)= & \frac{n_{i} V_{0}^{2}}{\hbar^{2}} \int_{0}^{2 \pi} d \theta \int_{0}^{\pi} \frac{1}{m}(\sin \varphi)^{2-m} d \varphi \int_{0}^{\Lambda} d k k^{2} \Gamma_{0} G_{0}^{m T}(\omega, k) \Gamma_{0} \\
= & \frac{2 \pi n_{i} V_{0}^{2}}{m \hbar^{2}} \int_{0}^{\pi}(\sin \varphi)^{2-m} d \varphi \\
& \frac{1}{12} \Lambda^{3}\left\{\frac{-4 \delta k_{z} \hbar v_{z}-\left[4 c_{3}^{2} m \sqrt{\frac{\xi^{2}(\Lambda \sin \varphi)^{2 m}}{c_{3}^{2}}+1_{2}} F_{1}\left(\frac{1}{2}, \frac{3}{2 m} ; 1+\frac{3}{2 m} ;-\frac{\xi^{2}(\Lambda \sin \varphi)^{2 m}}{c_{3}^{2}}\right)\right]}{\left[(m+3) \sqrt{c_{3}^{2}+\xi^{2}(k \sin \varphi)^{2 m}}\right]}\right. \\
& \left.-\frac{12 \sqrt{c_{3}^{2}+\xi^{2}(\Lambda \sin \varphi)^{2 m}}}{m+3}+3 \chi \hbar v_{z} \Lambda \cos \varphi-4 \mu\right\},
\end{aligned}
$$

where ${ }_{2} F_{1}(a, b ; c ; d)=\sum_{0}^{\infty} \frac{(a)_{n}(b)_{n}}{(c)_{n}} \frac{d^{n}}{n !}$ is the hypergeometric function. In such a 3D Dirac system, the Fermion spectral function in the absence of the quasiparticle scattering (noninteracting case) reads

$$
A_{0}^{m T}=\delta\left(\omega-\varepsilon^{m T}\right),
$$


where the locations of the sharp peaks are surely related to the above band dispersion. The longitudinal optical conductivity $\sigma_{z z}$ is finite unless when a pair of Weyl nodes tilted in parallel direction [60] in momentum space. The density of states for the 3D Dirac semimetal at halffilling can then be obtained as

$$
\begin{aligned}
D^{m T}= & \int \frac{d^{3} k}{(2 \pi)^{3}} A_{0}^{m T} \\
= & \int \frac{d^{3} k}{(2 \pi)^{3}} \delta\left(\omega-\left(\hbar v_{z} k_{z} \mp k^{m} \xi\right)\right) \\
\approx & \frac{1}{\pi} \int d k_{z} \int d k \frac{c}{c^{2}+\left(\hbar v_{z} k_{z} \mp k^{m} \xi\right)^{2}} \\
= & \text { onst. }_{1}+\text { const. }_{2} k \\
& -\frac{i k m}{2 \hbar v_{z}}\left[\left(\frac{\xi k m}{-\hbar v_{z} k_{z}+\xi k^{m}-i c}\right)^{-1 / m_{2}} F_{1}\left(-\frac{1}{m},-\frac{1}{m} ; \frac{m-1}{m} ; \frac{c-i \hbar v_{z} k_{z}}{i \xi k^{m}+c-i \hbar v_{z} k_{z}}\right)\right. \\
& \left.-\left(\frac{\xi k m}{-\hbar v_{z} k_{z}+\xi k^{m}+i c}\right)^{-1 / m}{ }_{2} F_{1}\left(-\frac{1}{m},-\frac{1}{m} ; \frac{m-1}{m} ; \frac{c+i \hbar v_{z} k_{z}}{-i \xi k^{m}+c+i \hbar v_{z} k_{z}}\right)\right] \\
& -\frac{k \tan ^{-1}\left(\frac{\xi k^{m}-\hbar v_{z} k_{z}}{c}\right)}{\hbar v_{z}},
\end{aligned}
$$

where $c$ is the small quantity used in the Lorentzian representation.

Base on the spectral function in the absence of the self-energy correction, the optical conductivity per Weyl node in the presence of nonzero Fermionic Matsubara frequency can be obtained by the Kubo formula

$$
\sigma_{z z}(\omega)=\frac{\operatorname{Im}_{z z}(\omega+i 0)}{\omega}
$$

then we have

$$
\begin{aligned}
\operatorname{Re} \sigma_{z z}(\omega) & =e^{2} \int_{-\infty}^{\infty} \frac{d \Omega}{2 \pi} \int \frac{d^{3} k}{(2 \pi)^{3}} \frac{N_{F}(\Omega-\mu)-N_{F}\left(\Omega^{\prime}-\mu\right)}{\Omega^{\prime}-\Omega+\omega+i 0} \operatorname{Tr}\left[\hat{v}_{z} A_{0}^{m T}\left(\Omega^{\prime}, k\right) \hat{v}_{z} A_{0}^{m T}(\Omega, k)\right] \\
& =\frac{e^{2}}{2 \omega+2 i 0} \int_{\mu-\omega}^{\mu} \frac{d \Omega}{2 \pi} \int \frac{d^{3} k}{(2 \pi)^{3}} v_{z}^{2}\left(\begin{array}{cc}
A_{1}^{m T}\left(\Omega^{\prime}\right)-A_{3}^{m T}\left(\Omega^{\prime}\right) & A_{2}^{m T^{\prime}}\left(\Omega^{\prime}\right)-A_{4}^{m T^{\prime}}\left(\Omega^{\prime}\right) \\
A_{2}^{m T}(\Omega)-A_{4}^{m T}(\Omega) & A_{1}^{m T^{\prime}}(\Omega)-A_{3}^{m T^{\prime}}(\Omega)
\end{array}\right),
\end{aligned}
$$

where we define $\Omega^{\prime}=\Omega+\omega, A^{m T^{\prime}}$ is the spectral function after optical transition. The velocity operator is $\hat{v}_{z}=v_{z} \sigma_{z}$. The components of the spectral function read

$$
\begin{aligned}
& A_{1}^{m T}(\Omega)=\pi\left[\delta\left(\Omega+\varepsilon^{m T}\right)+\delta\left(\Omega-\varepsilon^{m T}\right)\right], \\
& A_{3}^{m T}(\Omega)=\pi\left[\delta\left(\Omega+\varepsilon^{m T}\right)-\delta\left(\Omega-\varepsilon^{m T}\right)\right], \\
& A_{2}^{m T}(\Omega)=\pi\left[-\delta\left(\Omega+\varepsilon^{m T}\right)-\delta\left(\Omega-\varepsilon^{m T}\right)\right] \operatorname{sgn}\left(\varepsilon^{m T}\right), \\
& A_{4}^{m T}(\Omega)=\pi\left[-\delta\left(\Omega+\varepsilon^{m T}\right)+\delta\left(\Omega-\varepsilon^{m T}\right)\right] \operatorname{sgn}\left(\varepsilon^{m T}\right),
\end{aligned}
$$


then the above optical conductivity can be rewritten as

$$
\begin{aligned}
\operatorname{Re} \sigma_{z z}(\omega)= & \frac{e^{2}}{2 \omega+2 i 0} \int_{\mu-\omega}^{\mu} \frac{d \Omega}{2 \pi} \int \frac{d^{3} k}{(2 \pi)^{3}} 4 v_{z}^{2}\left(\begin{array}{cc}
\pi \delta\left(\Omega^{\prime}-\varepsilon^{m T}\right) & -\pi \delta\left(\Omega^{\prime}-\varepsilon^{m T^{\prime}}\right) \operatorname{sgn}\left(\varepsilon^{m T^{\prime}}\right) \\
-\pi \delta\left(\Omega-\varepsilon^{m T}\right) \operatorname{sgn}\left(\varepsilon^{m T}\right) & \pi \delta\left(\Omega-\varepsilon^{m T}\right)
\end{array}\right) \\
= & \frac{e^{2}}{2 \omega+2 i 0} \int_{\mu-\omega}^{\mu} \frac{d \Omega}{2 \pi} \int \frac{d^{3} k}{(2 \pi)^{3}} 4 v_{z}^{2} \pi^{2} \delta\left(\Omega-\varepsilon^{m T}\right)\left(\delta\left(\Omega^{\prime}-\varepsilon^{m T}\right)-\delta\left(\Omega^{\prime}-\varepsilon^{m T^{\prime}}\right) \operatorname{sgn}\left(\varepsilon^{m T}\right) \operatorname{sgn}\left(\varepsilon^{m T^{\prime}}\right)\right) \\
= & \frac{2 v_{z}^{2} \pi^{2} e^{2}}{\omega+i 0} \int_{\mu-\omega}^{\mu} \frac{d \Omega}{2 \pi} \frac{1}{m} \int_{0}^{2 \pi} d \theta \int_{0}^{\pi} d \varphi(\sin \varphi)^{2-m} \int_{0}^{\Lambda} d k \\
& k^{2} \delta\left(\Omega-\varepsilon^{m T}\right)\left(\delta\left(\Omega^{\prime}-\varepsilon^{m T}\right)-\delta\left(\Omega^{\prime}-\varepsilon^{m T^{\prime}}\right) \operatorname{sgn}\left(\varepsilon^{m T}\right) \operatorname{sgn}\left(\varepsilon^{m T^{\prime}}\right)\right)
\end{aligned}
$$

$\varepsilon^{m T^{\prime}}$ is the eigenenergy after the optical transition, and we note that, since we assume the hopping strength is isotropic and real (i.e., does not consider the chiral kind of hopping), the eigenenergy (dispersion) is real. Then the above integral can be solved by using the Lorentzian representation $\delta(x)=\frac{c}{\pi\left(c^{2}+x^{2}\right)}$ again where $c$ is a small quantity related to the quaisparticle scattering.

The particle spectral function including the disorder-induced self-energy effect reads 30 , 61, 76]

$$
A^{m T}(\Omega)=-\frac{1}{\pi} \frac{\left|\operatorname{Im} \Sigma^{D}(\Omega)\right|}{\left(\Omega-\operatorname{Re} \Sigma^{D}(\Omega)-\varepsilon^{m T}\right)^{2}+\left(\operatorname{Im} \Sigma^{D}(\Omega)\right)^{2}},
$$

which contains the informations about not only the dispersion but also the quasiparticle residue and the Fermion relaxation. In the presence of the screened long-range Coulomb interaction by the collective excitations in non-Fermi-liquid state (but with finite chemical potential), the above perturbed spectral function also related to the excitation damping, like the plasmon mode which damped into the particle-hole excitations due to the non-zero imaginary part of the polarization function (Bosonic self-energy) as we studied 62, 63, 64, 65]. The hole spectral function can be obtained simply as $-A^{m T}(\Omega)$.

\section{Conclusion}

In conclusion, we investigate the self-energy correction, symmetry, free energy, transverse optical conductivity of the 2D Dirac system in non-Fermi-liquid state. The non-Fermi-liquid behaviors of the 2D and 3D Dirac/Weyl systems with higher order dispersion are also studied and we found that the non-Fermi-liquid features remain even at finite chemical potential, and they are distinct from the Fermi-liquid picture and the conventional non-Fermi-liquid picture. In the presence of the impurity scattering, the Fermionic/Bosonic polaron formed by dressing the Fermion/Boson majority particles as widely found in ultracold Fermi gases 66, 67] and BEC [68], respectively, and they are also important in studying the perturbation effect within the contact potential (Dirac $\delta$-type impurity field) context. The many-body perturbation effect at the charge neutrality (the critical point) is related to the long-range Coulomb interaction and the coupling to the electronic critical mode 23 . Thus it is also interesting to considering the results in this paper to the polaron dynamics[78, 177]. In the presence of the gapless order parameter fluctuation, the Landau damping of the longitudinal excitations within RPA for the non-Fermi-liquid case are also discussed. For Weyl semimetal, the Chern number is given by $\chi m$, and the momentum $k_{z}$ along the diraction of the line connecting two Weyls nodes can be related to the topological phase transition, like the transition between trivial insulator phase and the quantum anomalous Hall phase. Further, since the higher order dispersion (with $m>1$ ), the DOS remains finite at zero-energy limit (charge neutrality), that makes the phase transition induced by interaction at this point more easy[26]. Further, for heavily tilted 
Weyl semimetal (i.e., the type-II where the tilt velocity is larger than the planar one and with unclosed electron pocket) [79, 80], the occupied states along the $k_{z}$ may create the particle-hole pairs[81] (particle-hole fluctuation), and physical observables in non-Fermi liquid picture like optical conductivity[21], can also be affected by the tilt.

\section{Appendix.A}

The Feynman diagrams of fermionic and bosonic self-energy are shown in Fig.4, where the external propagators are attached with the fermion loop. We note that, in the presence of electromagnetic field (like a gate voltage), the electromagnetic response can also be described by the Fermion loop as shown in Fig.4(b), with the electron propagator $G_{0}$ and external photon propagator [71, 17, 73].

For 2D Dirac system, the Coulomb induced fermi exchange self-energy for high-order dispersion reads

$$
\begin{aligned}
\Sigma_{C}(k, \omega) & =\int_{-\infty}^{\infty} \frac{d \Omega}{2 \pi} \int \frac{d^{2} q}{(2 \pi)^{2}} G_{0}(k+q, \omega+\Omega) \frac{2 \pi e^{2}}{\epsilon_{0}} \frac{1}{q-\frac{2 \pi e^{2}}{\epsilon_{0}} \Pi(q, \Omega)}, \\
\Pi(q, \Omega) & =g \int_{-\infty}^{\infty} \frac{d \omega}{2 \pi} \int \frac{d^{2} k}{(2 \pi)^{2}} \operatorname{Tr}\left[G_{0}(k+q, \omega+\Omega) G_{0}(k, \omega)\right]
\end{aligned}
$$

where the $1 / q$ in the denominator of the self-energy is the bare boson propagator $D_{0}$ which corresponds to the GV approximation, when the dielectric term (i.e., the dynamical polarization or density-density response function) is contained, the dressed boson propagator $D=$ $\frac{1}{q\left(1-\frac{2 \pi e^{2}}{\epsilon_{0} q} \Pi(q, \Omega)\right)}$ corresponds to the GW approximation, as despicted by the wave line in Fig.9(a). $\epsilon_{0}$ is the background dielectric constant. Here the bare propagator is approximated as $D_{0}=1 / q$, which is similar to the one $D_{0}^{\prime}=1 / q^{2}$ for three-dimensional case 69., however, the bare bosonic propagators may have more complex form in scaling analysis [70, 29].

Then we focus on the regime where $q, \Omega \rightarrow 0$. For approximated energy as stated above, we have $\varepsilon^{m}=\sqrt{\xi^{2} k^{2 m}+D^{2}}-\mu$ where $D$ denotes the band gap which is momentum-independent (due to the long-wave length and low-energy limit). Then the polarization function (densitydensity response or equivalently, the bosonic self-energy) at dynamical limit to order of $q^{2}$ reads [72, 74]

$$
\Pi(q, \Omega \rightarrow 0)=\frac{g v_{F}}{4 \pi \hbar \Gamma(2)} \frac{\xi^{2} k_{F}^{2}}{\sqrt{\xi^{2} k_{F}^{2}+D^{2}}} \frac{q^{2}}{\Omega^{2}},
$$

where $g$ is the degenerate number, $k_{F}=\sqrt{\mu^{2}-D^{2}}$, and the carrier concentration reads $n_{c}=$ $\frac{g \pi k_{F}^{2}}{4 \pi^{2} \Gamma(2)}$. This expression implies that the polarization is independent of the order $m$ in the long-wave length and low-energy limit, which will be verified in the following. Although this approximated polarization function inevitably introduce the pole at static limit $\Omega=0$, it will be proved to be correct (in following) for arbitary order $m$ in the limit of $q \rightarrow 0$ and $\Omega \rightarrow 0$. The long-wave length limit usually related to the Fermi liquid picture with $q \ll k_{F}[15$, which corresponds to the local density response. As can be seen, the above exchange self-energy does not contains the ladder correction. We can also easily see that there is a logarithmic divergence, which is consistent with the result of Ref.[12], such logarithmic divergence will appears also in the disorder-induced self-energy, but unlike the result in Hartree-Fock approximation [12, 11]: $\Sigma_{C} \sim \frac{e^{2}}{4 \epsilon_{0}} \ln \frac{\Lambda}{k}$ which is frequency-independent, the exchange self-energy here will becomes zero in the static limit due to the inclusion of dielectric term. 
Using the approximation up to order of $q^{2}$

$$
\begin{aligned}
\varepsilon_{k}-\varepsilon_{k^{\prime}} & =-\frac{\xi^{2} q k^{m}}{\varepsilon_{k}}-\frac{q^{2} D^{2} \xi^{2}}{2\left(\varepsilon_{k}^{2}\right)^{3 / 2}}+O\left(q^{3}\right), \\
\varepsilon_{k} \varepsilon_{k^{\prime}} & =\left(\varepsilon_{k}^{2}\right)+\xi^{2} q k^{m}+\frac{D^{2} \xi^{2} q^{2}}{2 \varepsilon_{k}^{2}}+O\left(q^{3}\right),
\end{aligned}
$$

the bosonic self-energy can be obtained by the Lehmann type formular as

$$
\Pi(q, \Omega)=2 \pi \sum_{s s^{\prime}} \int_{0}^{\Lambda} k g \frac{1}{2}\left(1+s s^{\prime} \cos b+\frac{D^{2}}{s \varepsilon_{k} s^{\prime} \varepsilon_{k^{\prime}}}\right) \frac{1}{\Omega+i 0+s \varepsilon_{k}-s^{\prime} \varepsilon_{k^{\prime}}} d k,
$$

which for intraband transition $\left(s s^{\prime}=1\right)$ equivalents to

$$
\begin{aligned}
\Pi(q, \Omega)= & \frac{0.00316629 g k^{2-3 m}\left(q \sqrt{\xi^{2} k^{2 m}}+k^{m}(\Omega+i \eta)\right)\left(4 k^{2 m}(-1+m)+q^{2} \sin ^{2} a\right)}{(-1+m)\left(\Omega^{2}-q^{2} \xi^{2}+2 i \Omega \eta-\eta^{2}\right)} \\
& +\left.\frac{0.00316629 g k^{2-3 m}\left(-q \sqrt{\xi^{2} k^{2 m}}+k^{m}(\Omega+i \eta)\right)\left(4 k^{2 m}(-1+m)+q^{2} \sin ^{2} a\right)}{(-1+m)\left(\Omega^{2}-q^{2} \xi^{2}+2 i \Omega \eta-\eta^{2}\right)}\right|_{0} ^{\Lambda},
\end{aligned}
$$

where the first term corresponds to the intra-conduction band transition and the second term corresponds to the intra-valence band transition, and we set $D=0$ and $\mu=0$ here for simplicity. From the this expression, we can know that in static limit $(\Omega=0)$ the total intraband polarization vanishes, and the polarization function obtained here in this approximation can not be applied to the case of linear dispersion $m=1$. The divergence of $\Pi(q, \Omega)$ can be seen from the first column of Fig.5, where we put it in the long-wave length limit first and then lowers the frequency to zero, which is in agree with Eq.(70). But we note that, when $q=\Omega=0$, $\Pi=0$. While for the interband part of the polarization $\left(s s^{\prime}=-1\right)$, the result is much more complicated than the intraband part (contains a series of hypergeometric functions), and an analytical solution is impossible to obtained until we set $m \geq 3$. That also reveals great difference compared to the ordinary Fermi liquid or the non-chiral electron gas. From Fig.6, we can see that the imaginary part of the polarization in static limit is still nearly independent of the order $m$, and the rest panels show that, the imaginary part is nonzero only around the point where $\Omega=v_{F} k$, which is due to the vanishing particle-hole continuum region, and we find the linewidth of the peak increases with the increase of $m$.

The self-energy and spectral function about the intra-conduction band transition are presented in the Fig.7 and Fig.8, respectively. Note that the self-energy here is calculated in long-wavelength limit $q \rightarrow 0$, which can be recognized as $q \ll t$ in the lattice model where $t$ is the hopping amplitude. The spectral function in Fig.8 is calculated according to the retarded self-energy (Fig.6-7) based on the dressed boson propagator. We can see that the width of imaginary part of self-energy decrease with the increase of order $m$, and their locations also shift toward right with the increase of electron frequency, but the shift distance decreases with the increase of $m$. Similar phenomenon can be seen from the spectral functions which all have finite peak width, and that is different to the Fermi liquid in normal metals where the shape of spectral function is close to the $\delta$ function due to the weak interacting nature. Except the static case, we find that the peaks in spectral function are symmetrical. Then base on the spectral function, the DOS as well as the occupation probability can be obtained by integrate over the momentum $k$ and $\omega$, respectively.

\section{References}

[1] Li X, Wang J R, Liu G Z. Phase transition with trivial quantum criticality in an anisotropic Weyl semimetal[J]. Physical Review B, 2018, 97(18): 184508. 
[2] Vekhter I, Chubukov A V. Non-Fermi-liquid behavior in itinerant antiferromagnets[J]. Physical review letters, 2004, 93(1): 016405.

[3] Shiranzaei M, Fransson J, Cheraghchi H, et al. Nonlinear spin susceptibility in topological insulators[J]. Physical Review B, 2018, 97(18): 180402.

[4] Chen-Huan Wu. Complex polaron formed on surface of two-dimensional lattice system in weak coupling regime. arXiv: 1906.06359

[5] Sagi Y, Drake T E, Paudel R, et al. Breakdown of the fermi liquid description for strongly interacting fermions $[\mathrm{J}]$. Physical review letters, 2015, 114(7): 075301.

[6] Christensen R S, Levinsen J, Bruun G M. Quasiparticle properties of a mobile impurity in a Bose-Einstein condensate[J]. Physical review letters, 2015, 115(16): 160401.

[7] Wu C H. Electronic properties of the parabolic Dirac system[J]. Physics Letters A, 2019, 383(15): 17951805.

[8] Honerkamp C, Salmhofer M, Furukawa N, et al. Breakdown of the Landau-Fermi liquid in two dimensions due to umklapp scattering[J]. Physical Review B, 2001, 63(3): 035109.

[9] Jia X, Zhang S, Sankar R, et al. Anomalous acoustic plasmon mode from topologically protected states[J]. Physical review letters, 2017, 119(13): 136805.

[10] Attaccalite C, Wirtz L, Lazzeri M, et al. Doped graphene as tunable electron-phonon coupling material[J]. Nano letters, 2010, 10(4): 1172-1176.

[11] Elias D C, Gorbachev R V, Mayorov A S, et al. Dirac cones reshaped by interaction effects in suspended graphene[J]. Nature Physics, 2011, 7(9): 701.

[12] Vafek O, Case M J. Renormalization group approach to two-dimensional Coulomb interacting Dirac fermions with random gauge potential[J]. Physical Review B, 2008, 77(3): 033410.

[13] Wu F, Hwang E, Sarma S D. Phonon-induced giant linear-in-T resistivity in magic angle twisted bilayer graphene: Ordinary strangeness and exotic superconductivity[J]. Physical Review B, 2019, 99(16): 165112.

[14] Culcer D. Linear response theory of interacting topological insulators[J]. Physical Review B, 2011, 84(23): 235411.

[15] Raghu S, Chung S B, Qi X L, et al. Collective modes of a helical liquid[J]. Physical review letters, 2010 , 104(11): 116401.

[16] Jafari S A, Baskaran G. Equations-of-motion method for triplet excitation operators in graphene[J]. Journal of Physics: Condensed Matter, 2012, 24(9): 095601.

[17] Jalali-Mola Z, Jafari S A. Electromagnetic modes from Stoner enhancement: Graphene as a case study[J]. Journal of Magnetism and Magnetic Materials, 2019, 471: 220-235.

[18] Yazyev O V, Katsnelson M I. Magnetic correlations at graphene edges: basis for novel spintronics devices[J]. Physical Review Letters, 2008, 100(4): 047209.

[19] Zhang S, Wei T, Guan J, et al. Enhanced Superconducting State in FeSe/SrTiO 3 by a Dynamic Interfacial Polaron Mechanism[J]. Physical review letters, 2019, 122(6): 066802.

[20] Shvonski A, Kong J, Kempa K. Plasmon-polaron of the topological metallic surface states[J]. Physical Review B, 2019, 99(12): 125148.

[21] Ahn S, Mele E J, Min H. Optical conductivity of multi-Weyl semimetals[J]. Physical Review B, 2017, 95(16): 161112 .

[22] Liu W E, Hankiewicz E M, Culcer D. Quantum transport in Weyl semimetal thin films in the presence of spin-orbit coupled impurities[J]. Physical Review B, 2017, 96(4): 045307.

[23] Han S E, Lee C, Moon E G, et al. Emergent Anisotropic Non-Fermi Liquid at a Topological Phase Transition in Three Dimensions[J]. Physical review letters, 2019, 122(18): 187601. 
[24] Cao Y, Fatemi V, Fang S, et al. Unconventional superconductivity in magic-angle graphene superlattices[J]. Nature, 2018, 556(7699): 43.

[25] Sentef M A. Light-enhanced electron-phonon coupling from nonlinear electron-phonon coupling[J]. Physical Review B, 2017, 95(20): 205111.

[26] Nandkishore R, Levitov L. Electron interactions in bilayer graphene: Marginal Fermi liquid and zero-bias anomaly[J]. Physical Review B, 2010, 82(11): 115431.

[27] Adibi E, Jafari S A. Strong-coupling approach to Mott transition of massless and massive Dirac fermions on honeycomb lattice[J]. Physical Review B, 2016, 93(7): 075122.

[28] Jafari S A. Collective excitations and the nature of Mott transition in undoped gapped graphene[J]. Journal of Physics: Condensed Matter, 2012, 24(30): 305601.

[29] González J, Guinea F, Vozmediano M A H. Marginal-Fermi-liquid behavior from two-dimensional Coulomb interaction[J]. Physical Review B, 1999, 59(4): R2474.

[30] Wang J R, Liu G Z, Zhang C J. Breakdown of Fermi liquid theory in topological multi-Weyl semimetals[J]. Physical Review B, 2018, 98(20): 205113.

[31] Hague J P. Tunable graphene band gaps from superstrate-mediated interactions[J]. Physical Review B, 2011, 84(15): 155438.

[32] Grover T, Sheng D N, Vishwanath A. Emergent space-time supersymmetry at the boundary of a topological phase[J]. Science, 2014, 344(6181): 280-283.

[33] Jian S K, Jiang Y F, Yao H. Emergent spacetime supersymmetry in 3D Weyl semimetals and 2D Dirac semimetals[J]. Physical review letters, 2015, 114(23): 237001.

[34] Onida G, Reining L, Rubio A. Electronic excitations: density-functional versus many-body Greens-function approaches[J]. Reviews of Modern Physics, 2002, 74(2): 601.

[35] Motome Y, Furukawa N. Disorder effect on spin excitation in double-exchange systems[J]. Physical Review B, 2005, 71(1): 014446 .

[36] Van Otterlo A, Wagenblast K H, Fazio R, et al. Response of Josephson-junction arrays near the quantum phase transition[J]. Physical Review B, 1993, 48(5): 3316.

[37] Ziegler K. Delocalization of 2D dirac fermions: The role of a broken supersymmetry[J]. Physical review letters, 1998, 80(14): 3113.

[38] Khaetskii A. Nonexistence of intrinsic spin currents[J]. Physical review letters, 2006, 96(5): 056602.

[39] Camacho-Guardian A, Goldman N, Massignan P, et al. Dropping an impurity into a Chern insulator: a polaron view on topological matter[J]. arXiv preprint arXiv:1811.00563, 2018.

[40] Yin S, Jian S K, Yao H. Chiral tricritical point: a new universality class in Dirac systems[J]. Physical review letters, 2018, 120(21): 215702.

[41] Mross D F, McGreevy J, Liu H, et al. Controlled expansion for certain non-Fermi-liquid metals[J]. Physical Review B, 2010, 82(4): 045121.

[42] Senthil T. Critical Fermi surfaces and non-Fermi liquid metals[J]. Physical Review B, 2008, $78(3)$ : 035103.

[43] von Löhneysen H. Non-Fermi-liquid behaviour in the heavy-fermion system[J]. Journal of Physics: Condensed Matter, 1996, 8(48): 9689.

[44] Streda P. Theory of quantised Hall conductivity in two dimensions[J]. Journal of Physics C: Solid State Physics, 1982, 15(22): L717.

[45] Tahir M. Electrical and optical transport properties of single layer WSe2[J]. Physica E: Low-dimensional Systems and Nanostructures, 2018, 97: 184-190.

[46] Wu C H. Geometrical structure and the electron transport properties of monolayer and bilayer silicene near the semimetal-insulator transition point in tight-binding model[J]. arXiv preprint arXiv:1805.00350, 2018. 
[47] Burkov A A, Balents L. Weyl semimetal in a topological insulator multilayer[J]. Physical review letters, 2011, 107(12): 127205.

[48] Shiranzaei M, Parhizgar F, Fransson J, et al. Impurity scattering on the surface of topological-insulator thin films[J]. Physical Review B, 2017, 95(23): 235429.

[49] Park S, Min H, Hwang E H, et al. Diluted magnetic Dirac-Weyl materials: Susceptibility and ferromagnetism in three-dimensional chiral gapless semimetals[J]. arXiv preprint arXiv:1804.10867, 2018.

[50] Scholz A, Stauber T, Schliemann J. Plasmons and screening in a monolayer of MoS 2[J]. Physical Review B, 2013, 88(3): 035135 .

[51] Jalali-Mola Z, Jafari S A. Tilt-induced kink in the plasmon dispersion of two-dimensional Dirac electrons[J]. Physical Review B, 2018, 98(19): 195415.

[52] Jalali-Mola Z, Jafari S A. Kinked plasmon dispersion in borophene-borophene and borophene-graphene double layers[J]. Physical Review B, 2018, 98(23): 235430.

[53] Yang B J, Nagaosa N. Classification of stable three-dimensional Dirac semimetals with nontrivial topology[J]. Nature communications, 2014, 5: 4898.

[54] Xiong J, Kushwaha S K, Liang T, et al. Evidence for the chiral anomaly in the Dirac semimetal Na3Bi[J]. Science, 2015, 350(6259): 413-416.

[55] Wu Y, Wang L L, Mun E, et al. Dirac node arcs in PtSn 4[J]. Nature Physics, 2016, $12(7): 667$.

[56] Moors K, Zyuzin A A, Zyuzin A Y, et al. Disorder-driven exceptional lines and Fermi ribbons in tilted nodal-line semimetals[J]. arXiv preprint arXiv:1810.03191, 2018.

[57] Bernardet K, Pzmndi F, Batrouni G G. Disorder averaging and finite-size scaling[J]. Physical review letters, 2000, 84(19): 4477.

[58] John S. Strong localization of photons in certain disordered dielectric superlattices[J]. Physical review letters, 1987, 58(23): 2486.

[59] Isobe H, Nagaosa N. Renormalization group study of electromagnetic interaction in multi-Dirac-node systems[J]. Physical Review B, 2013, 87(20): 205138.

[60] Das K, Agarwal A. Linear magneto-chiral transport in tilted type-I and type-II Weyl Semimetals[J]. arXiv preprint arXiv:1808.03646, 2018.

[61] Briggs S, Mason B A, Leburton J P. Self-consistent polaron scattering rates in quasi-one-dimensional structures[J]. Physical Review B, 1989, 40(17): 12001.

[62] Wu C H. Dynamical polarization and the optical response of silicene and related materials[J]. Results in Physics, 2018, 11: 665-673.

[63] Wu C H. Electronic transport and dynamical polarization in bilayer silicene-like systems[J]. Results in Physics, 2018.

[64] Wu C H. Interband and intraband transition, dynamical polarization and screening of the monolayer and bilayer silicene in low-energy tight-binding model[J]. Indian J. Phys. 2018.

[65] Wu C H. Dynamical currentcurrent correlation in two-dimensional parabolic Dirac systems[J]. Physics Letters A, 2018.

[66] Schirotzek A, Wu C H, Sommer A, et al. Observation of Fermi polarons in a tunable Fermi liquid of ultracold atoms[J]. Physical review letters, 2009, 102(23): 230402.

[67] Kohstall C, Zaccanti M, Jag M, et al. Metastability and coherence of repulsive polarons in a strongly interacting Fermi mixture[J]. Nature, 2012, 485(7400): 615.

[68] Li W, Sarma S D. Variational study of polarons in Bose-Einstein condensates[J]. Physical Review A, 2014, 90(1): 013618 . 
[69] Goswami P, Chakravarty S. Quantum criticality between topological and band insulators in $3+1$ dimensions[J]. Physical review letters, 2011, 107(19): 196803.

[70] Metlitski M A, Sachdev S. Quantum phase transitions of metals in two spatial dimensions. I. Ising-nematic order[J]. Physical Review B, 2010, 82(7): 075127.

[71] Isobe H, Nagaosa N. Renormalization group study of electromagnetic interaction in multi-Dirac-node systems[J]. Physical Review B, 2013, 87(20): 205138.

[72] Sachdeva R, Thakur A, Vignale G, et al. Plasmon modes of a massive Dirac plasma, and their superlattices[J]. Physical Review B, 2015, 91(20): 205426.

[73] Pozo Ó, Ferreiros Y, Vozmediano M A H. Anisotropic fixed points in Dirac and Weyl semimetals[J]. Physical Review B, 2018, 98(11): 115122.

[74] Sarma S D, Hwang E H. Collective modes of the massless Dirac plasma[J]. Physical review letters, 2009, 102(20): 206412.

[75] Wang J, Culcer D. Suppression of the Kondo resistivity minimum in topological insulators[J]. Physical Review B, 2013, 88(12): 125140.

[76] Hassaneen K S A. Spectral functions of nuclear matter using self-consistent Greens function approach based on three-body force[J]. The European Physical Journal Plus, 2018, 133(11): 484.

[77] Wu C H. Attractive polaron in a Dirac/Weyl system within the ladder approximation[J]. arXiv preprint arXiv:1812.04833, 2018.

[78] Wu C H. Attractive fermi polaron in a semi-Dirac system within ladder approximation[J]. arXiv preprint arXiv:1901.07881, 2019.

[79] Farajollahpour T, Faraei Z, Jafari S A. Solid-state platform for space-time engineering: The $8 \mathrm{P} \mathrm{m} \mathrm{m} \mathrm{n}$ borophene sheet[J]. Physical Review B, 2019, 99(23): 235150.

[80] Baskaran G, Jafari S A. Gapless spin-1 neutral collective mode branch for graphite[J]. Physical review letters, 2002, 89(1): 016402.

[81] Zubkov M A, Lewkowicz M. The type II Weyl semimetals at low temperatures: Chiral anomaly, elastic deformations, zero sound[J]. Annals of Physics, 2018, 399: 26-52. 
Fig.1
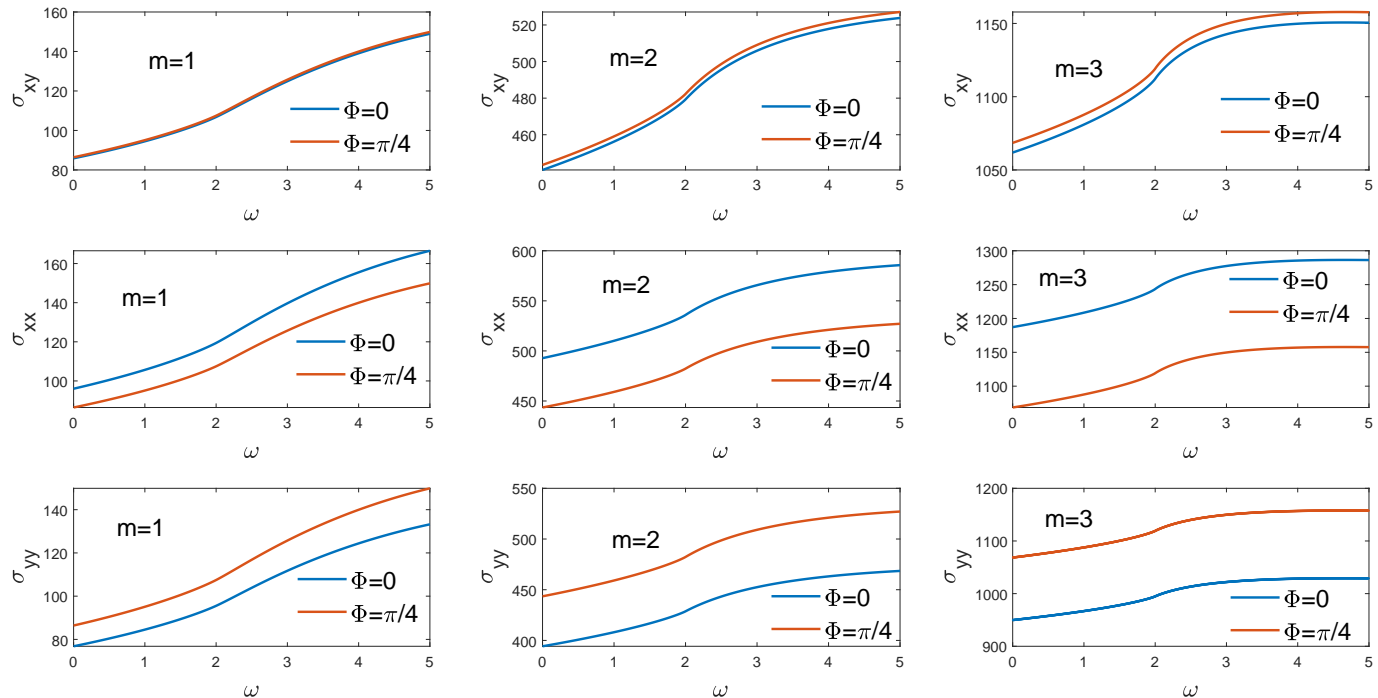

Figure 1: Opticl conductivity in 3D Dirac system for interband scattering from up-spin to down-spin. The first row is the transverse conductivity $\sigma_{x y}$. The second and third rows are the longitudinal conductivities $\sigma_{x x}$ and $\sigma_{y y}$. The colums from left to right correspond to the order $m=1,2$ and 3 . The vertical axis is in unit of $\frac{i \hbar e^{2}}{(2 \pi)^{3}}$. Here we set $c_{1}=c_{2}=1, \xi=2$. Since we consider the low-temperature limit and clean limit, and set chemical potential.as $\mu=0$, the optical conductivities here don't show a gap due to the Pauli blocking. 
Fig. 2
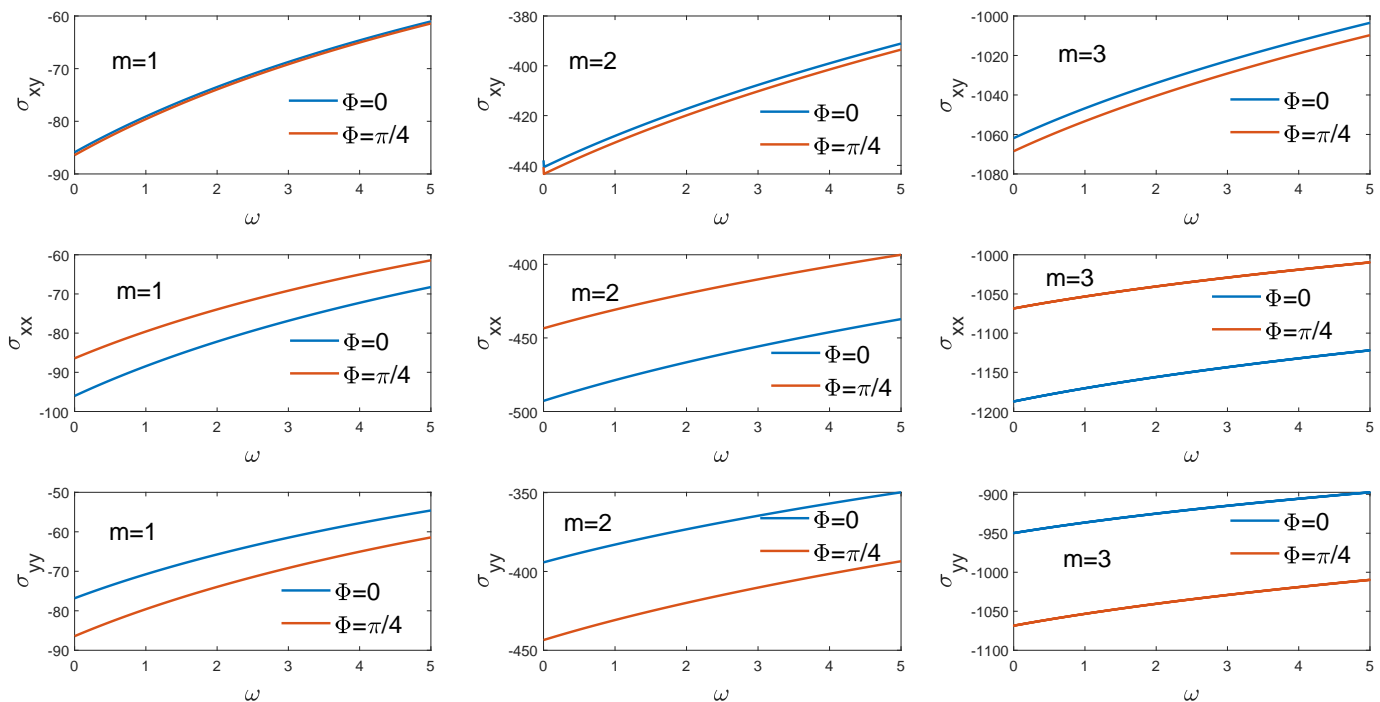

Figure 2: The same as Fig.2 but for interband scattering from down-spin to up-spin. 
Fig.3
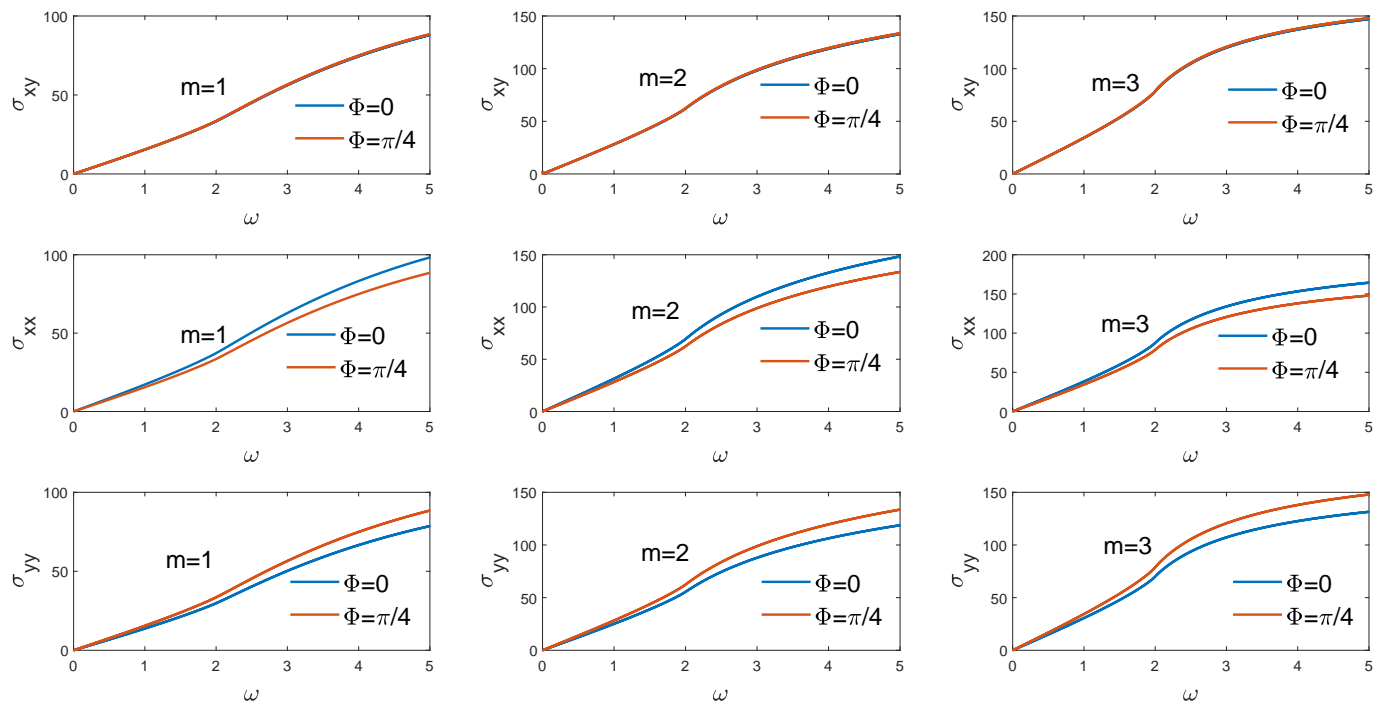

Figure 3: The total optical conductivity where we consider the interband scatterings from down-spin to up-spin and up-spin to down-spin. 
Fig.4

(a)

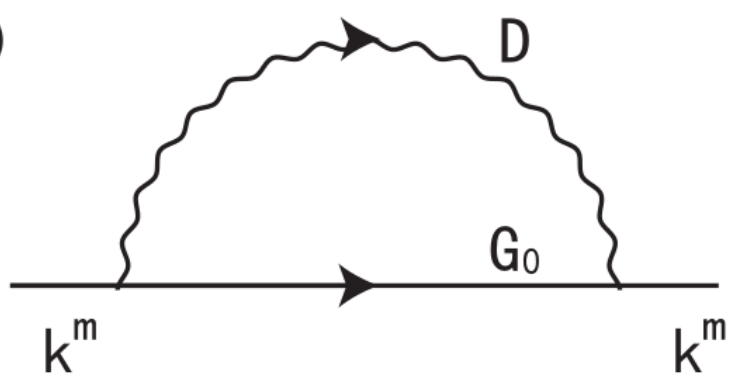

(b)

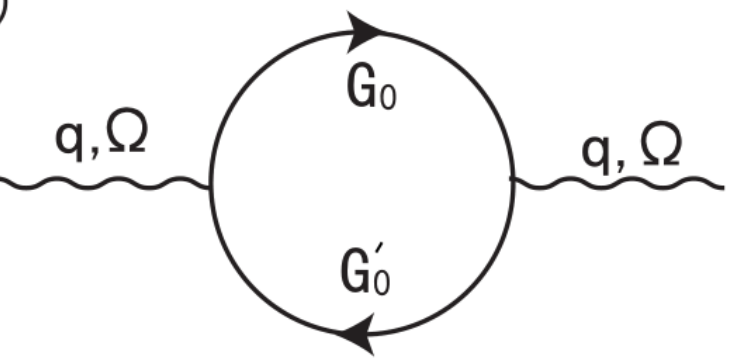

Figure 4: (a) Fermion self-energy with the dressed scalar potential propagator $D$. (b) Boson self-emergy with scattering momentum and frequency $q, \Omega$.

Fig.5
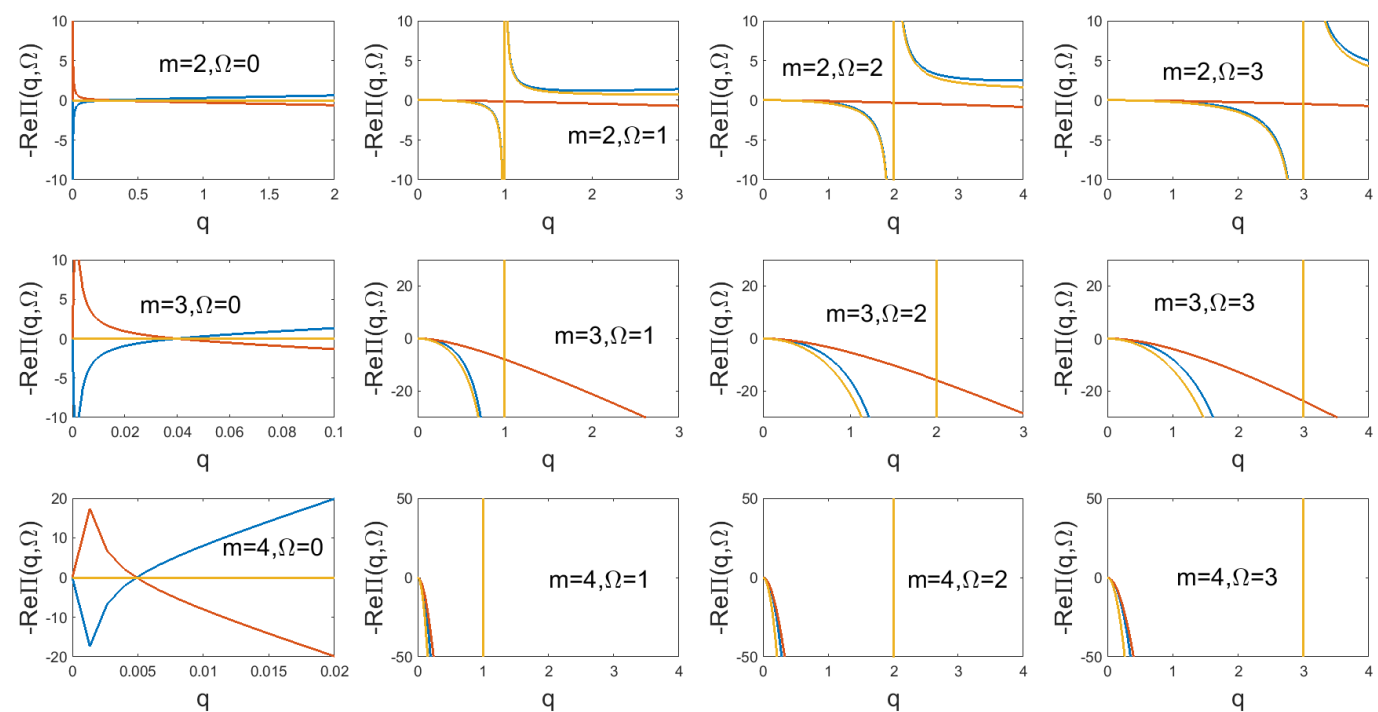

Figure 5: Intra-conduction band part (blue) intra-valence band part (red) of the polarization function, and their summation (yellow). The vertical axis is in unit of $2 \pi$ and we set $a=\pi / 4, \xi=1$. 
Fig.6 Fig.7
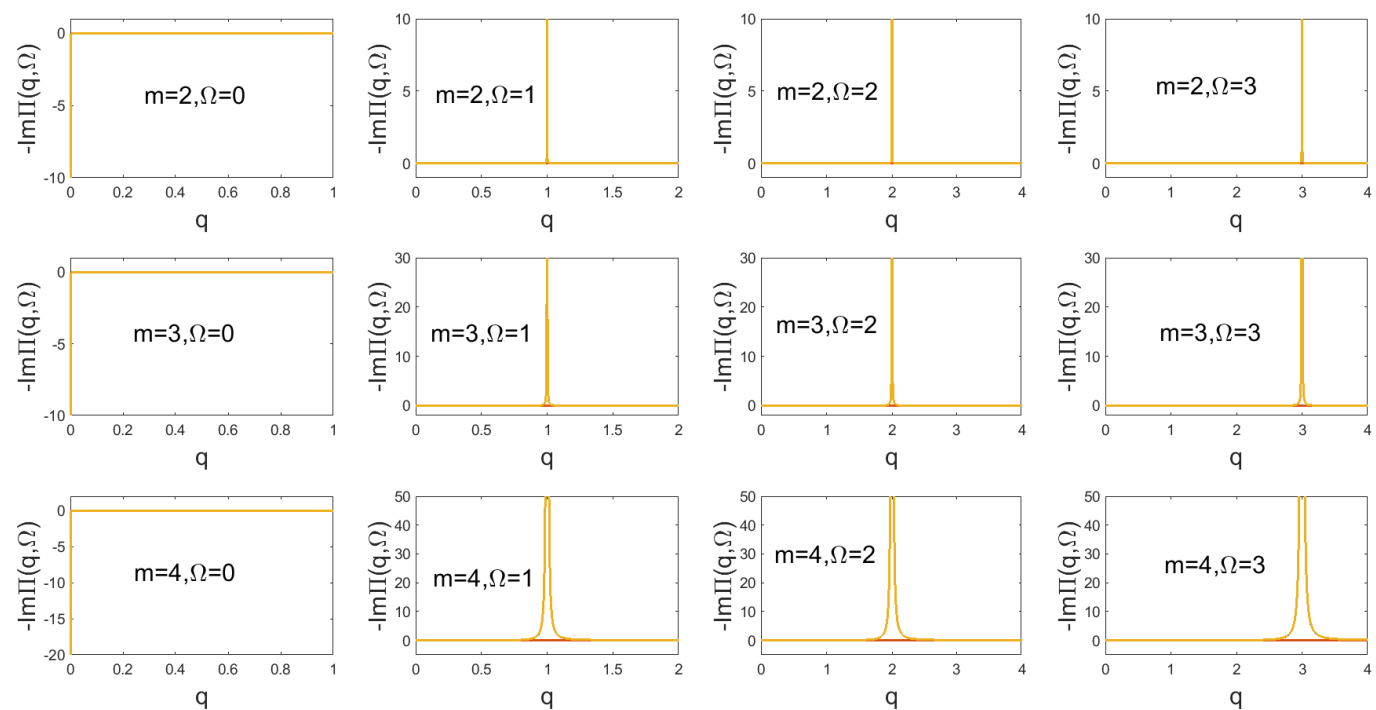

Figure 6: Imaginary part of the polarization function corresponds to Fig.5. We can see that the imaginary part of the polarization (and the spectral function) only contributed by the intra-conduction band part (blue lines).
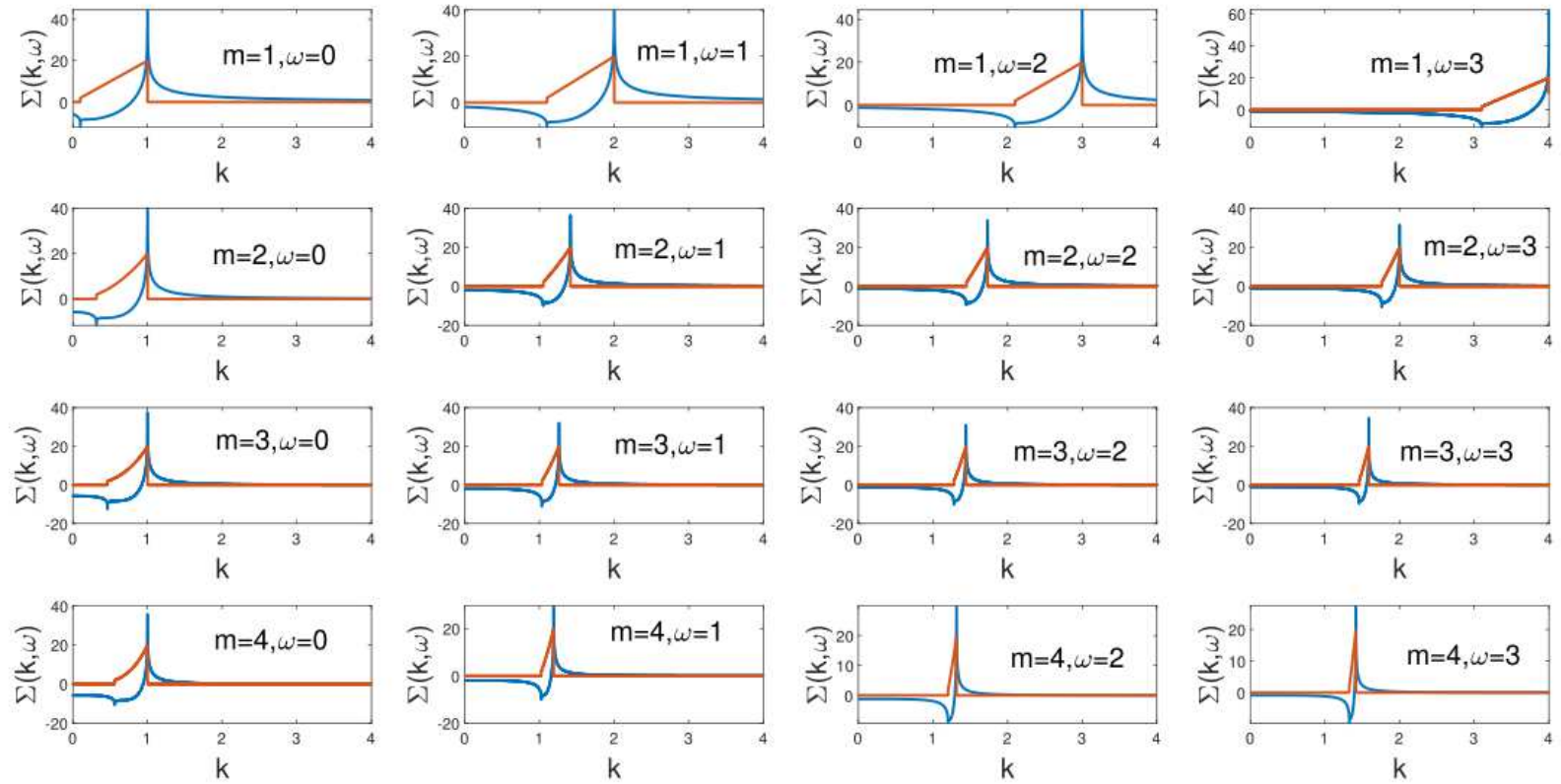

Figure 7: Self-energy calculated by the intra-conduction band polarization. The blue line and red line correspond to the real part and imaginary part, respectively. 
Fig.8
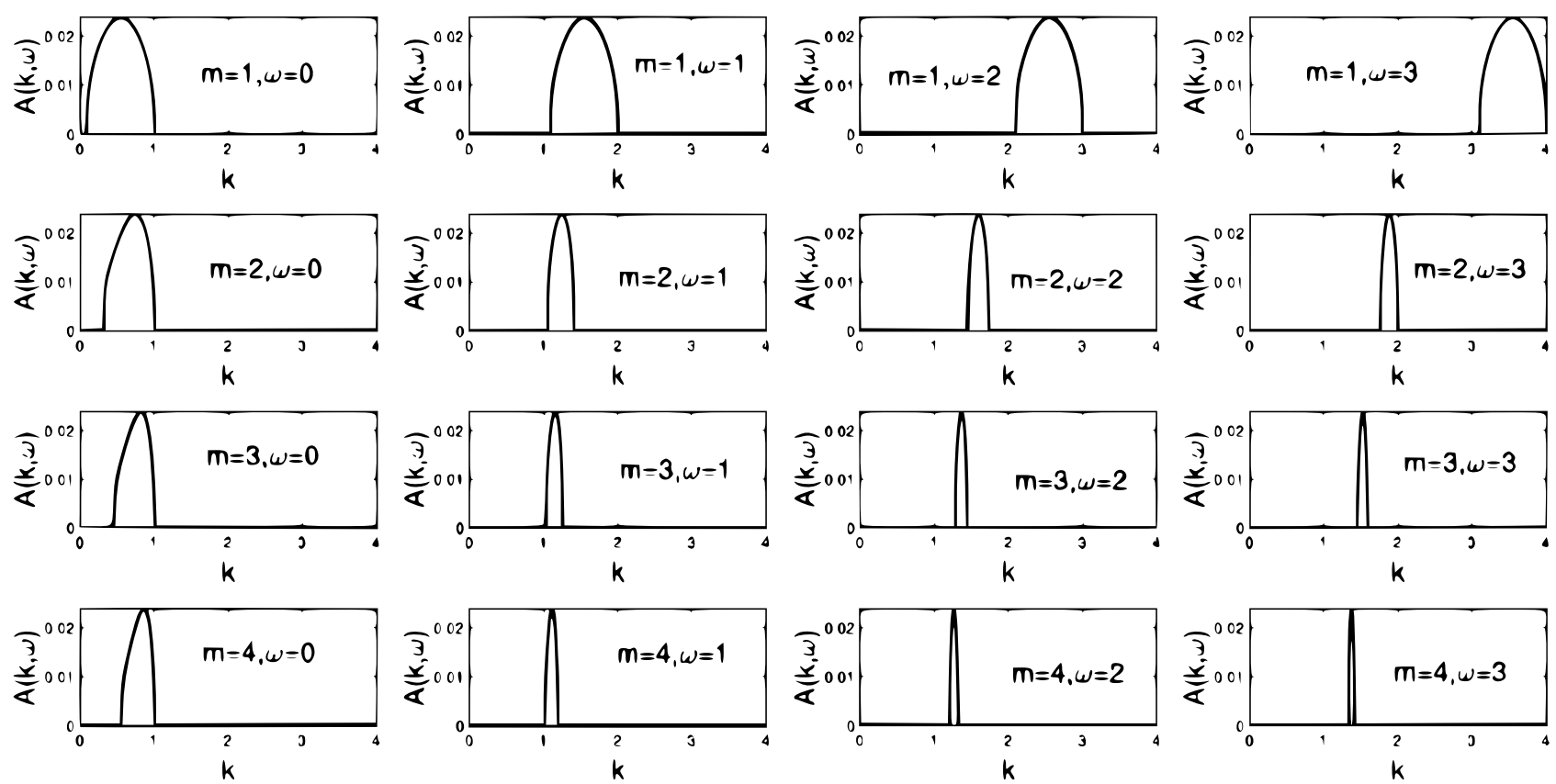

Figure 8: Particle spectral function calculated by the intra-conduction band polarization. 